\title{
Equilibrium morphology of misfit particles in elastically stressed solids under
}

\section{chemo-mechanical equilibrium conditions}

Xujun Zhao $^{a, 1}$, Stéphane P.A. Bordas ${ }^{c, d, 2}$ and Jianmin $\mathrm{Qu}^{a, b, 3}$

${ }^{a}$ Department of Mechanical Engineering, Northwestern University, Evanston, IL USA 60208.

${ }^{b}$ Department of Civil and Environmental Engineering, Northwestern University, Evanston, IL USA 60208.

${ }^{c}$ University of Luxembourg, Research Unit in Engineering, Computational Mechanics Group, Luxembourg.

${ }^{d}$ Cardiff School of Engineering, Institute of Mechanics and Advanced Materials Theoretical, Applied and Computational Mechanics, Cardiff University, The Parade, Cardiff CF24 3AA, Wales, UK

\begin{abstract}
This paper studies the effects of chemical, elastic and interfacial energies on the equilibrium morphology of misfit particles due to phase separation in binary alloys under chemo-mechanical equilibrium conditions. A continuum framework that governs the chemo-mechanical equilibrium of the system is first developed using a variational approach by treating the phase interface as a sharp interface endowed with interfacial excess energy. An extended finite element method (XFEM) in conjunction with the level set method is then developed to simulate the behaviors of

${ }^{1}$ Present address: Mathematics and Computer Science Division, Argonne National Laboratory, Lemont, IL USA 60439. Email:xzhao99@gmail.com

2 stephane.bordas@uni.lu

${ }^{3}$ Corresponding author at: j-qu@ @orthwestern.edu (Jianmin Qu)
\end{abstract}


the coupled chemo-mechanical system. The coupled chemo-mechanics model together with the numerical techniques developed here provides an efficient simulation tool to predict the equilibrium morphologies of precipitates in phase separate alloys.

Keywords: Chemo-mechanical coupling; Equilibrium morphology; Phase transformation; Extended finite element method (XFEM); Level set method;

\section{Introduction}

When a solid solution is in a non-equilibrium state, which may be caused by external factors such as heat treatment or other perturbations, its internal microstructure will gradually evolve into other states that are more thermodynamically stable. During this process, atoms tend to rearrange their spatial positions to reduce the total system free energy. Consequently, second phase particles may form, grow, and gradually change their size, shape, morphology, and even chemical composition. For example, particles in coherent two-phase systems such as $\gamma / \gamma^{\prime}$ in Ni-base alloys and $\mathrm{A} 2 / \mathrm{DO}_{3}$ in Fe-base alloys not only show different shapes from spheres to cuboid with round corners, but also show different particle sizes and highly spatial correlated distributions (Conley et al., 1989; Doi, 1996; Fahrmann et al., 1995). More complex morphological changes have also been observed experimentally, such as splitting of one single particle into a group of small particles and merging of neighboring particles to form long plate-like large particles (Ardell and Nickolson, 1966; Doi et al., 1984; Ma and Ardell, 2007; Miyazaki et al., 1982). 
Since the thermomechanical properties of such phase separated alloys, to a large extent, depend on the microstructure of the separated phases, the ability to control the morphological evolution of the phases enables the design and processing of high performance alloys. Therefore, it is of paramount importance to understand the morphological development of phase separations resulting from diffusional phase transformations. Furthermore, effective and efficient simulation tools are needed to predict the stable morphology of the separated phases.

From a thermodynamic point of view, the equilibrium morphology corresponds to the minimum of total system free energy. The morphological evolution of such a system is governed by the competition of three different energies, chemical free energy, elastic strain energy and interfacial excess energy. The chemical free energy is only related to the temperature and chemical composition of each component, irrespective of the actual structure of each phase. The elastic strain energy and interfacial excess energy are caused by the lattice mismatch between precipitate and matrix phases and the existence of a particle-matrix interface, respectively. For a stress-free system, chemical free energy alone determines the total volume fraction of each equilibrium phase and the interfacial energy tends to minimize the total interface areas in order to reduce the interfacial energy. The equilibrium shape in this situation can be easily obtained. However, if the misfit strain is present, the elastic strain energy will play an important role in shaping the precipitate morphology. Experimental observations show that elastic strain energy can significantly influence the particle shape, size, as well as spatial distributions during phase transformations (Doi, 1996; Fahrmann et al., 1995).

Due to numerical difficulties in tracking evolving interfaces and in dealing with the interface 
boundary conditions, diffuse interface models, or phase field methods, are usually used to study this class of problems involving morphological evolution. Phase field methods assume that the field quantities are continuously changing from one phase to another within a narrow region, so that the interface is actually modeled as an interphase layer with a finite thickness. The main advantage of the diffuse interface model is that there is no need to track the interface and impose the interface boundary conditions because there is no material interface involved, which provides greater convenience for numerical implementations. Phase field models have been successfully used for microstructural evolution problems, such as solidification (Braun et al., 1994; Wang and Sekerka, 1996), solid-solid phase transformations (Lou and Bassani, 2008; Maraldi et al., 2011; Wang and Khachaturyan, 1995; Zaeem and Mesarovic, 2010), dislocations (Rodney et al., 2003) and many others. For more applications of phase field models, the readers are referred to recent review papers (Chen, 2002; Moelans et al., 2008; Singer-Loginova and Singer, 2008).

One drawback of the phase field method is that the computational grid spacing must be smaller than the interface thickness in order to resolve the diffuse interface. Three-dimensional atomic probe microscope observations have shown that the thickness of precipitate-matrix interface is typically less than $2 \mathrm{~nm}$ (Isheim and Seidman, 2004), which means that the phase field grid needs be finer than $2 \mathrm{~nm}$ near the interface. This obviously increases the computational costs significantly. Another drawback of the phase field method is that the system free energy has to be formulated in terms of several order parameters. These parameters usually do not have clear physical interpretations and are difficult to determine.

An alternative approach is the sharp interface model, in which different phases are separated 
by a geometric dividing interface and the governing equations are solved in each phase under specific boundary conditions. Simulations of microstructural evolution based on sharp interface models mainly use the boundary integral method (BIM). For example, Voorhees and coworkers studied the morphological evolution of precipitate particles in an elastically anisotropic but homogeneous system using BIM (Su and Voorhees, 1996a, b; Thornton et al., 2001; Voorhees et al., 1992). Those works were later extended to inhomogeneous elastic systems (Jou et al., 1997; Leo et al., 2000). However, the conventional boundary integral based methods encounter great difficulties when dealing with topological changes during microstructural evolution. For instance, particle merging and splitting phenomena, which are common for particles during the phase transformations, cannot be captured easily in those BIM-based approaches. Recently, Duddu et al. (Duddu et al., 2011) revisited this problem based on the same mathematical model using a combined extended finite element method (XFEM) and level set method (LSM), in which the geometry of interface was implicitly defined by the level set function so that topological changes could be easily handled.

It should be pointed out that the theoretical models used in the above sharp-interface simulations are based on the assumption that the chemical and mechanical fields are only one-way coupled in that the misfit (eigen-) strain and elastic deformation are independent of composition, so that they can be computed separately. In our previous work (Zhao et al., 2013a; Zhao et al., 2013b), a sharp interface model incorporating the Gurtin-Murdoch interface elasticity effects was developed to study the equilibrium morphology of misfit particles in general elastic anisotropic solids and implemented using a hybrid smoothed XFEM/LSM, in 
which the chemical free energy was neglected based on the late-stage coarsening assumption, so the particle size consequently remained constant during evolution.

In the present work, we present a two-phase continuum model for a generally anisotropic elastic system with misfit particles incorporating the chemical free energy, elastic strain energy and interfacial energy, which allows precipitate particles to change both their sizes and shapes under the conditions that the system satisfies chemical equilibrium, mechanical equilibrium and mass conservation. We employ a coherent sharp interface model to implicitly describe the geometry of the precipitate-matrix interface using the level set method. The interface is endowed with an interfacial excess energy, which includes two parts: the interfacial chemical free energy and the interfacial elastic energy. The interfacial elastic energy is described using the Gurtin-Murdoch interface elasticity model (Gurtin and Murdoch, 1975). The chemo-mechanically coupled equilibrium equations and the interfacial equilibrium conditions are derived by minimizing the total system free energy using a variational approach, and the kinetics of phase transformations is derived using a time derivative approach. These equations are then solved via a coupled nonlinear extended finite element method (XFEM), which allows the phase interface to be completely independent of the underlying meshes, so that remeshing is avoided when the interface evolves. The driving force for the interface to evolve in the direction of reducing the total system energy is also derived and connected to the level set evolution equation.

The paper is arranged as follows. First, the problem of phase separation in binary alloys is formulated in Sec. 2, where the constitutive equations and system energies are introduced. In 
addition, the governing equations are derived by minimizing the total system free energy. Also discussed in this section is the kinetics of the interface evolution. In Sec. 3, the hybrid XFEM/LSM method is developed, and numerical results from the XFEM/LSM simulations are presented in Sec. 4. Finally, Sec. 5 gives a brief summary and some concluding remarks.

\section{Problem Formulation}

We consider a binary solid $\mathrm{A}_{c} \mathrm{~B}_{1-c}$ containing species $\mathrm{A}$ and species $\mathrm{B}$, where $c$ is the molar fraction (concentration) of species A. We assume that the solid in consideration can be represented by the network model of Larche and Cahn (Larche and Cahn, 1973). Specifically, we assume that the lattice sites of species B form a network within which species A can move (diffuse). This allows the definition of a displacement and hence a strain of the solid. We further assume that the solid can segregate into two phases, the matrix phase occupying the volume $V_{m}$ and the precipitate phase occupying the volume $V_{p}$. The total volume of the solid is thus $V=V_{m}+V_{p}$. The precipitate-matrix interface denoted by $\Gamma$ is assumed coherent. The interaction between the precipitates and dislocations is also neglected (Chen et al., 2010). Finally, we assume that the system is aged at a constant temperature for long enough time so chemical equilibrium is achieved under isothermal conditions. We note that the alloy $\mathrm{A}_{c} \mathrm{~B}_{1-c}$ may be either a crystalline or an amorphous solid. In addition, the derivation below can be easily extended to multi-phase and multi-species solids.

To be definitive, let us assume that an initial distribution of $\mathrm{A}$ is given throughout $V$. If this initial distribution does not form stable phases, diffusion will take place driven by the

\footnotetext{
4 Throughout this paper, the term "volume" is used to mean a domain with its shape as well as the narrow meaning of volume.
} 
concentration gradient and stresses. After long enough time has elapsed, both chemical and mechanical equilibria will be achieved when the alloy phase-segregates into precipitate and matrix phases. Our objective in this paper is to develop a theoretical and computational framework that enables the prediction of such a stable phase segregation under both mechanical and chemical equilibria.

\subsection{Constitutive equations}

We assume that the deformation is small so that the binary solid can be considered linearly elastic. In addition, the insertion of species A into species B causes deformation of the network formed by species B, which causes an eigenstrain, or a compositional strain. Consequently, the elastic strain energy density function $w$ is a function of the total strain and the concentration of species A, and can be written as

$$
w(c, \boldsymbol{\varepsilon})=\frac{1}{2}\left(\boldsymbol{\varepsilon}-\boldsymbol{\varepsilon}^{c}\right): \mathbf{C}:\left(\boldsymbol{\varepsilon}-\boldsymbol{\varepsilon}^{c}\right)
$$

where $\boldsymbol{\varepsilon}$ is the total elastic strain and $\mathbf{C}$ is the elastic stiffness tensor. $\boldsymbol{\varepsilon}^{c}$ represents the compositional strain. Without loss of generality, we assume that the compositional strain is given by

$$
\boldsymbol{\varepsilon}^{c}(c)=\left(c-c_{r}\right) \boldsymbol{\eta}
$$

where $c_{r}$ is the stoichiometric concentration and $\boldsymbol{\eta}$ is the compositional strain coefficient. In many cases of practical interests, $\boldsymbol{\eta}$ is dilatational, i.e., $\boldsymbol{\eta}=\eta \mathbf{I}$, where $\eta$ is the coefficient of compositional expansion (CCE) (Cui et al., 2012; Swaminathan et al., 2007) and I is the second order identity tensor. The selection of $c_{r}$ can be somewhat arbitrary as far as finding stable phase separation is concerned. Some authors, e.g., (Lou and Bassani, 2008), use $c_{r}=c_{m}$ where $c_{m}$ is the equilibrium concentration of the matrix phase under stress-free conditions. In 
this paper, $c_{r}$ will be taken as the average concentration $c_{0}$. Once $c_{r}$ is identified, we consider $\mathrm{A}_{c_{r}} \mathrm{~B}_{1-c_{r}}$ as the initial stress-free configuration (the reference configuration). Any change in the concentration of A will cause compositional strain.

Further, the Cauchy stress tensor and the elastic strain tensor are given by

$$
\boldsymbol{\sigma}=\mathbf{C}:\left(\boldsymbol{\varepsilon}-\boldsymbol{\varepsilon}^{c}\right), \quad \boldsymbol{\varepsilon}=\frac{1}{2}\left[\nabla \mathbf{u}+(\nabla \mathbf{u})^{T}\right]
$$

Note that $\mathbf{u}$ gives the displacement of material particles located at $\mathbf{x}$ in the reference configuration.

Consequently, the total strain energy of the solid occupying $V$ can be written as

$$
F_{\text {elastic }}=\int_{V} w(c, \boldsymbol{\varepsilon}) d V=\frac{1}{2} \int_{V}\left(\boldsymbol{\varepsilon}-\boldsymbol{\varepsilon}^{c}\right): \mathbf{C}:\left(\boldsymbol{\varepsilon}-\boldsymbol{\varepsilon}^{c}\right) d V
$$

In addition to the strain energy, there is also chemical free energy in the solid. Let $f(c)$ be the chemical free energy density, the total chemical free energy of the solid contained in the volume $V$ can then be written as,

$$
F_{\text {chem }}=\int_{V} f(c) d V
$$

In the situation of phase separation, the chemical free energy is often defined by a non-convex double-well function of concentration $c$ (Cahn and Hilliard, 1958; Porter and Easterling, 1992).

$$
f(c)=c f_{A}+(1-c) f_{B}+\Omega c(1-c)+k_{B} T[c \log c+(1-c) \log (1-c)],
$$

where $f_{A}$ and $f_{B}$ are the chemical free energy densities of pure species A and pure species B, respectively, and $\Omega$ is the interaction energy density, which is independent of temperature and composition. The parameters $k_{B}$ and $T$ are the standard Boltzmann's constant and absolute temperature, respectively. The first two linear terms in (6) represent the contributions from pure 
species A and pure species B when they are fully separated. These two terms are usually neglected in many of the phase field models. The last two terms are the change in chemical free energy density caused by mixing species A and species B, according to the Flory-Huggins model (Flory, 1941; Huggins, 1941).

However, in order to avoid numerical difficulties caused by the logarithmic terms, polynomial approximations are usually adopted in most literatures (Lou and Bassani, 2008; Tonks et al., 2012; Wodo and Ganapathysubramanian, 2011; Zaeem and Mesarovic, 2010). In this work, we use the following double-well function

$$
f(c)=\min \left\{f_{m}(c), f_{p}(c)\right\} \quad,
$$

where $f_{m, p}(c)$ is a convex free energy function for the $m$ and $p$ phases, respectively,

$$
f_{m, p}(c)=A_{m, \mathrm{p}}\left(c-c_{m, p}\right)^{2}+B_{m, p}
$$

In the above, $A_{m, p}$ and $B_{m, p}$ are positive constants intrinsic to the materials, and $c_{m, p}$ is the equilibrium concentration for the $m$ and $p$ phases, respectively. In other words, $c_{m, p}$ corresponds to the local minimums of $f_{m, p}(c)$ under stress-free conditions. The above expression can reproduce accurately the double-well potential function used in phase field models (Wang and Khachaturyan, 1995). In addition, it is straightforward to extend it to multiple-well potential functions if multiple metastable phases exist for the alloy.

Analogous to the bulk energy, we assume that the interfacial excess free energy includes not only the elastic part due to the interface deformation, but also the chemical part associated with the interfacial excess concentration $c_{s}$. Consequently, the total interfacial excess free energy can be written as 


$$
F_{\text {surf }}=\int_{\Gamma}\left[\gamma\left(c_{s}, \boldsymbol{\varepsilon}^{s}\right)+f_{s}\left(c_{s}\right)\right] d S
$$

where $\gamma$ is the elastic interfacial excess strain energy density and $f_{s}$ is the chemical interfacial excess energy density. Both $\gamma$ and $f_{s}$ may depend on the interfacial excess concentration $c_{s}$. Specifically, ignoring the effects of curvature (Gao et al., 2014) and the effects of transverse deformation (Dingreville and $\mathrm{Qu}, 2008$ ) on the elastic interfacial excess energy, we adopt the following elastic interfacial excess energy,

$$
\gamma=\gamma_{0}+\boldsymbol{\tau}^{s}:\left(\boldsymbol{\varepsilon}^{s}-\boldsymbol{\varepsilon}^{*}\right)+\frac{1}{2}\left(\boldsymbol{\varepsilon}^{s}-\boldsymbol{\varepsilon}^{*}\right): \mathbf{C}^{s}:\left(\boldsymbol{\varepsilon}^{s}-\boldsymbol{\varepsilon}^{*}\right),
$$

where $\boldsymbol{\varepsilon}^{s}$ is the total interfacial strain, and $\boldsymbol{\varepsilon}^{*}$ is the interfacial eigenstrain so that $\boldsymbol{\varepsilon}^{s}-\boldsymbol{\varepsilon}^{*}$

gives the interfacial elastic strain. Here and in the followings, field quantities with a superscript $s$ are associated with the interface. For example, $\boldsymbol{\tau}^{s}$ is the interface residual stress and $\mathbf{C}^{s}$ denotes the interface elastic stiffness tensor. For future reference, the interfacial compliance tenor will be denoted by $\mathbf{S}^{s}$. The parameter $\gamma_{0}$ represents the intrinsic interfacial excess elastic energy density at in the absence of any elastic deformation, i.e., $\boldsymbol{\varepsilon}^{s}-\boldsymbol{\varepsilon}^{*}=\mathbf{0}$. Note that $\gamma_{0}, \boldsymbol{\tau}^{s}$ and $\mathbf{C}^{s}$ are the properties of the interface, and can be computed from the constituent materials using molecular dynamic simulations (Dingreville and Qu, 2009).

For coherent interfaces, the interfacial strain is defined as the projection of bulk strain tensor on the tangential plane,

$$
\boldsymbol{\varepsilon}^{s}=\frac{1}{2}\left[\left(\nabla_{s} \mathbf{u}\right)+\left(\nabla_{s} \mathbf{u}\right)^{T}\right]
$$

where $\nabla_{s} \mathbf{u}=\nabla \mathbf{u} \cdot \mathbf{P}$ is the surface gradient of displacement, $\mathbf{P}=\mathbf{I}-\mathbf{n} \otimes \mathbf{n}$ is the tangential projection tensor, $\otimes$ represents a dyad, and $\mathrm{n}$ is a unit vector normal to the interface.

The interfacial compositional (eigen-) strain can be written as 


$$
\boldsymbol{\varepsilon}^{*}=\left(c_{s}-c_{s}^{0}\right) \boldsymbol{\eta}^{s}
$$

where $c_{s}^{0}$ is a stoichiometric interfacial concentration, and $\boldsymbol{\eta}^{s}$ is the compositional strain coefficient of the interface. The corresponding interfacial stress can be obtained from the Shuttleworth equation,

$$
\boldsymbol{\sigma}^{s}=\frac{\partial \gamma}{\partial\left(\boldsymbol{\varepsilon}^{s}-\boldsymbol{\varepsilon}^{*}\right)}=\frac{\partial \gamma}{\partial \boldsymbol{\varepsilon}^{s}}=\boldsymbol{\tau}^{s}+\mathbf{C}^{s}:\left(\boldsymbol{\varepsilon}^{s}-\boldsymbol{\varepsilon}^{*}\right)
$$

The interfacial energy and the interfacial stress are generally neglected in traditional continuum mechanics. However, the influence of these effects is much more pronounced for small scale microstructures where the ratio of interfacial energy density to the bulk modulus is comparable to the characteristic length of material elements (Duan et al., 2005; Zhao and Qu, 2012; Zhao and Rajapakse, 2009).

\subsection{System free energy}

The total free energy of the binary solid may include chemical energy, elastic strain energy and interfacial energy,

$$
F=F_{\text {chem }}+F_{\text {elastic }}+F_{\text {surf }}=\int_{V}[f(c)+w(c, \mathbf{u})] d V+\int_{\Gamma}\left[\gamma\left(c_{s}, \mathbf{u}\right)+f_{s}\left(c_{s}\right)\right] d S .
$$

In addition, we assume that the alloy is under constant temperature (isothermal), and has no mass and temperature exchange with its surroundings (isolated). Therefore, the total mass must be conserved,

$$
\int_{V} c d V+\int_{\Gamma} c_{s} d S=V c_{0}
$$

This constraint may be released by introducing a Lagrangian multiplier $\bar{\mu}$ via

$$
L=F-\bar{\mu}\left[\int_{V} c d V+\int_{\Gamma} c_{s} d S-V c_{0}\right] \quad .
$$




\subsection{Chemical and mechanical equilibriums}

For simplicity, we assume the outer surface $\partial V$ is traction-free, i.e.,

$$
\left.\boldsymbol{\sigma} \cdot \mathbf{m}\right|_{\mathbf{x} \in \partial V}=\mathbf{0}
$$

where $\mathbf{m}$ is the outward normal of $\partial V$. Thus, under chemical and mechanical equilibria, the first variation of the total system free energy (16) must vanish,

$$
\delta L=\delta F_{\text {chem }}+\delta F_{\text {elastic }}+\delta F_{\text {suf }}-\delta\left(\bar{\mu}\left[\int_{V} c d V+\int_{\Gamma} c_{s} d S-V c_{0}\right]\right)=0 .
$$

We note that $L$ is a functional of three basic fields quantities, the solute concentration $c$ in the bulk, the solution concentration $c_{s}$ at the interface, and the displacement vector $\mathbf{u}$. It then follows from (5) that,

$$
\delta F_{\text {chem }}=\int_{V} \mu \delta c d V
$$

where $\mu=\partial f / \partial c$ is the chemical potential under stress-free condition. Note that both the solute concentration $c$ and the chemical potential $\mu$ are discontinuous across the phase interface.

For the elastic strain energy,

$$
\delta F_{\text {elastic }}=\int_{V} \boldsymbol{\sigma}: \nabla \delta \mathbf{u} d V-\int_{V} \boldsymbol{\sigma}: \boldsymbol{\eta} \delta c d V+\frac{1}{2} \int_{V} \boldsymbol{\sigma}: \delta \mathbf{S}: \boldsymbol{\sigma} d V
$$

where $\mathbf{S}$ is the elastic compliance tensor so that $\boldsymbol{\varepsilon}-\boldsymbol{\varepsilon}^{c}=\mathbf{S}: \boldsymbol{\sigma}$. Upon using the Green's theorem and integration by parts, Eq. (20) can be further written as

$$
\delta F_{\text {elastic }}=-\int_{V} \delta \mathbf{u} \cdot(\nabla \cdot \boldsymbol{\sigma}) d V-\int_{\Gamma} \delta \mathbf{u} \cdot \boldsymbol{\sigma} \cdot \mathbf{n} d S-\int_{V}\left(\boldsymbol{\sigma}: \boldsymbol{\eta}-\frac{1}{2} \boldsymbol{\sigma}: \frac{\partial \mathbf{S}}{\partial c}: \boldsymbol{\sigma}\right) \delta c d V
$$

where the double brackets stand for the jump of the quantity across the interface,

$$
\cdot=(\cdot)^{+}-(\cdot)^{-} \text {. }
$$

Similarly, the variation of interfacial excess energy yields 


$$
\delta F_{\text {surf }}=-\int_{\Gamma}\left(\nabla_{s} \cdot \boldsymbol{\sigma}^{s}\right) \cdot \delta \mathbf{u} d S+\int_{\Gamma}\left[\mu_{s}-\boldsymbol{\sigma}^{s}: \boldsymbol{\eta}^{s}\right] \delta c_{s} d S
$$

where $\mu_{s}=\partial f_{s} / \partial c_{s}$ may be viewed as the interfacial chemical potential under stress-free

conditions. In deriving the above, we have used (13) and the fact that $\int_{\Gamma} \nabla_{s} \cdot\left(\boldsymbol{\sigma}^{S} \cdot \delta \mathbf{u}\right) d S=0$ for any closed surface/contour $\Gamma$, see (Gurtin and Murdoch, 1975). Finally, the variation of $L$ gives

$$
\delta\left(\bar{\mu}\left[\int_{V} c d V+\int_{\Gamma} c_{s} d S-V c_{0}\right]\right)=\bar{\mu} \int_{V} \delta c d V+\bar{\mu} \int_{\Gamma} \delta c_{s} d V+\left[\int_{V} c d V+\int_{\Gamma} c_{s} d S-V c_{0}\right] \delta \bar{\mu}
$$

Substituting equations (19)-(23) into (18), we obtain

$$
\begin{aligned}
& \int_{V}\left[\mu-\left(\boldsymbol{\sigma}: \boldsymbol{\eta}-\frac{1}{2} \boldsymbol{\sigma}: \frac{\partial \mathbf{S}}{\partial c}: \boldsymbol{\sigma}\right)-\bar{\mu}\right] \delta c d V-\int_{V}(\nabla \cdot \boldsymbol{\sigma}) \cdot \delta \mathbf{u} \cdot d V-\left[\int_{V} c d V+\int_{\Gamma} c_{s} d S-V c_{0}\right] \delta \bar{\mu} \\
& -\int_{\Gamma}\left(\nabla_{s} \cdot \boldsymbol{\sigma}^{S}+\boldsymbol{\sigma} \cdot \mathbf{n}\right) \cdot \delta \mathbf{u} d S+\int_{\Gamma}\left(\mu_{s}-\boldsymbol{\sigma}^{S}: \boldsymbol{\eta}^{S}-\bar{\mu}\right) \delta c_{s} d S=0
\end{aligned}
$$

This gives the weak form of the governing equation for chemo-mechanical equilibrium. The corresponding Euler equations are

$$
\begin{aligned}
& \int_{V} c d V+\int_{\Gamma} c_{s} d S=V c_{0}, \\
& \nabla \cdot \boldsymbol{\sigma}=0 \text { in } V, \\
& \bar{\mu}=\mu-\boldsymbol{\sigma}: \boldsymbol{\eta}+\frac{1}{2} \boldsymbol{\sigma}: \frac{\partial \mathbf{S}}{\partial c}: \boldsymbol{\sigma} \text { in } V \\
& \boldsymbol{\sigma} \cdot \mathbf{n}+\nabla_{s} \cdot \boldsymbol{\sigma}^{s}=0, \quad \bar{\mu}=\mu_{s}-\boldsymbol{\sigma}^{s}: \boldsymbol{\eta}^{s} \text { on } \Gamma
\end{aligned}
$$

It is seen that the Lagrangian multiplier $\bar{\mu}$ is nothing but stress-dependent chemical potential derived in (Larche and Cahn, 1973, 1978) for an isothermal system. Equations (27) indicate that $\bar{\mu}$ is uniform throughout the entire system including the interface. This does not mean that the chemical potential itself is uniform as the case discussed in (Cahn, 1980). In particular, $\mu$ and $\mu_{s}$ are not necessarily the same. Further, the first of (28) gives interface mechanical equilibrium, which is the generalized Young-Laplace equation derived in (Povstenko, 1993) when the interface stress is present. The second of (28) introduces an interface 
stress-dependent interfacial chemical potential.

The above equations (26) - (28) constitute the basic governing equations for the mechanical and chemical equilibria for a two-phase mass-conserved system. It is seen that the elastic deformation and chemical composition are fully coupled not only in the bulk, but also at the interface. Furthermore, due to the nonlinearity of the chemical potential, the system of coupling equations is nonlinear.

\subsection{Kinetics of the interface}

The governing equations derived above are for a fixed volume of the precipitate. In other words, the solute concentration $c$ and the displacement $\mathbf{u}$ that satisfy (25) - (28) minimizes the system free energy under chemo-mechanical for the given precipitate volume $V_{p}$. Our goal in this paper, however, is to find the $V_{p}$ that minimizes the system free energy under chemo-mechanical equilibrium. This can be achieved by varying $V_{p}$, for example, by trial and error until the system free energy becomes the smallest. Instead of trial and error, a more systematic way of finding the optimal $V_{p}$ is to introduce a mapping that transforms $V_{p}$ to a new configuration $\bar{V}_{p}$,

$$
\overline{\mathbf{x}}(\mathbf{x}, t)=\mathbf{x}+t \mathbf{v}(\mathbf{x})
$$

where $t$ is a parameter to indicate the process of transformation, and $\mathbf{v}(\mathbf{x})=d \overline{\mathbf{x}}(\mathbf{x}, 0) / d t$ represents the amount of displacement at location $\mathbf{x}$ per unit change of $t$. Under this mapping, the precipitate volume $V_{p}$ can be viewed as a function of $t$, so can the matrix volume $V_{m}$.

Consequently, the system free energy $F$ given by (14) becomes a function of $t$. In other words, as 
$t$ changes, $F$ will change. Since we are looking for an optimal $V_{p}$ that minimizes $F$, we may

select $\mathbf{v}(\mathbf{x})$ such that the change of $F$ with respect to $t$ is always negative. This guarantees that transforming $V_{p}$ to $\bar{V}_{p}$ decreases the system free energy, so eventually the system free energy is minimized.

To evaluate the rate of change of $F$ with respect to $t$, we can take advantage of the Reynolds transport theorem by interpreting $t$ as time and $\mathbf{v}(\mathbf{x})$ as velocity (Arora, 1993). For a volume integral

$$
I=\int_{V} g(\mathbf{x}, t) d V
$$

where the domain of integration changes with $t$, the Reynolds transport theorem states

$$
\dot{I}=\int_{V}[\dot{g}+g \nabla \cdot \mathbf{v}] d V=\int_{V}\left[g^{\prime}+\nabla \cdot(g \mathbf{v})\right] d V=\int_{V} g^{\prime} d V+\oint_{\partial V} g \mathbf{v} \cdot \mathbf{n} d S
$$

where an over dot indicates the so called material derivative in continuum mechanics, for

example,

$$
\dot{g}=g^{\prime}+(\nabla g) \cdot \mathbf{v}
$$

In the above, a prime means the local rate of change $g^{\prime} \equiv \partial g / \partial t$, i.e., the change in $g$ with respect to $t$ as if $\mathbf{v}=\mathbf{0}$. The last term of (31) is obtained by using the divergence theorem where $\partial V$ is the surface of $V$ and $\mathbf{n}$ is its outward unit normal vector.

The transport theorem for an integral on a closed surface ${ }^{5}$

$$
I_{S}=\oint_{S} g(\mathbf{x}, t) d S
$$

is a little more involved because material derivative is not well-defined if the surface is not a

\footnotetext{
${ }^{5}$ For the purpose of this paper, we consider only closed surface. The transport theorem works also for open surfaces, albeit the expressions is a little more complex.
} 
material surface. Further, $g^{\prime} \equiv \partial g / \partial t$ may no longer be on the surface (Cermelli et al., 2005). One way to overcome these difficulties is to extend the definition of the integrand to a three-dimensional neighborhood of the surface. A geometrically natural method of smoothly extending a surface field $g(\mathbf{x}, t)$, at each time $t$, to a three-dimensional region containing the surface is obtained by requiring that $g(\mathbf{x}, t)$ be constant on normal lines, where a normal line at time $t$ is a line through a point $\mathbf{x}$ on $S$ parallel to its unit normal vector $\mathbf{n}=\mathbf{n}(\mathbf{x}, t)$. With such understanding, the transport theorem for the surface integral defined in (33) is given by (Arora, 1993; Cermelli et al., 2005),

$$
\dot{I}_{S}=\oint_{S}(\dot{g}-g \kappa \mathbf{v} \cdot \mathbf{n}) d S=\oint_{S}\left[g^{\prime}+(\nabla g) \cdot \mathbf{n}-g \kappa \mathbf{v} \cdot \mathbf{n}\right] d S
$$

where $\kappa=-\nabla_{s} \cdot \mathbf{n}$ is the total (i.e. twice the mean) curvature.

Having laid out the mathematical tools, we can now restate our original objective of finding the optimal $V_{p}$ that minimizes the system free energy $F$. By taking advantage of the concept of material derivative and the transport theorem outline above, our objective can be alternatively stated as finding $\mathbf{v}(\mathbf{x})$ so that $\dot{F}<0$, i.e., an infinitesimal change to the precipitate volume must reduce the system free energy. In what follows, we will first calculate $\dot{F}$. Then, use $\dot{F}<0$ to solve for $\mathbf{v}(\mathbf{x})$.

Making use of (31) the above formulae of material derivatives together with the Green's theorem, the time variations for each part of energy functional can be readily derived. First, the material derivative of the chemical free energy (5) is

$$
\dot{F}_{c h e \overline{\bar{m}}} \int_{V} \mu \dot{c} d V \oint_{\Gamma} \quad \boldsymbol{N} \mathbf{n} \quad .
$$

Similarly, 


$$
\dot{F}_{\text {elastic }}=-\int_{\Gamma} \mathbf{n} \cdot \boldsymbol{\sigma} \cdot \dot{\mathbf{u}} d S-\int_{\Gamma} \mathbf{n} \cdot \llbracket \Sigma^{e} \rrbracket \cdot \mathbf{v} d S-\int_{V}\left(\boldsymbol{\sigma}: \boldsymbol{\eta}-\frac{1}{2} \boldsymbol{\sigma}: \frac{\partial \mathbf{S}}{\partial c}: \boldsymbol{\sigma}\right) c^{\prime} d V
$$

where $\Sigma^{e}=w \mathbf{I}-\boldsymbol{\sigma} \cdot \nabla \mathbf{u}$ is the classical Eshelby energy momentum tensor. In deriving the above, we have used the fact that $\nabla \cdot \boldsymbol{\sigma}=0$ in $V$, see (26). Next, we calculate $\dot{F}_{\text {surf }}$ using (34),

$$
\begin{aligned}
\dot{F}_{\text {surf }}= & -\int_{\Gamma} \dot{\mathbf{u}} \cdot \nabla_{s} \cdot \boldsymbol{\sigma}^{S} d S+\int_{\Gamma}\left(\mu_{s}-\boldsymbol{\sigma}^{s}: \boldsymbol{\eta}^{s}\right) \dot{c}_{s} d S \\
& +\int_{\Gamma}\left[\mathbf{v} \cdot \nabla_{S} \cdot\left(\boldsymbol{\sigma}^{S} \cdot \nabla_{S} \mathbf{u}\right)-\left(\gamma+f_{s}\right) \kappa \mathbf{v} \cdot \mathbf{n}\right] d S
\end{aligned}
$$

In arriving at the above, we have use the fact that $\bar{\mu}=\mu_{s}-\boldsymbol{\sigma}^{s}: \boldsymbol{\eta}^{s}$ on the interface, see the second of (28).

The sum of (35) - (37) gives

$$
\begin{aligned}
\dot{F} & =\int_{V_{m}}\left[\mu_{m}-\left(\boldsymbol{\sigma}: \boldsymbol{\eta}^{c}-\frac{1}{2} \boldsymbol{\sigma}: \frac{\partial \mathbf{S}}{\partial c}: \boldsymbol{\sigma}\right)\right] c^{\prime} d V+\int_{V_{p}}\left[\mu_{p}-\left(\boldsymbol{\sigma}: \boldsymbol{\eta}^{c}-\frac{1}{2} \boldsymbol{\sigma}: \frac{\partial \mathbf{S}}{\partial c}: \boldsymbol{\sigma}\right)\right] c^{\prime} d V \\
& +\int_{\Gamma}\left(\mu_{s}-\boldsymbol{\sigma}^{s}: \boldsymbol{\eta}^{s}\right) \dot{c}_{s} d S \\
& -\int_{\Gamma}\left\{\mathbf{n} \cdot\left[\llbracket \Sigma^{e}+f \mathbf{I} \rrbracket+\left(\gamma+f_{s}\right) \kappa \mathbf{I}\right]-\nabla_{s} \cdot\left(\boldsymbol{\sigma}^{s} \cdot \nabla_{s} \mathbf{u}\right)\right\} \cdot \mathbf{v} d S
\end{aligned}
$$

The interface equilibrium condition (28) has been used in deriving (38). Further, application of (31) and (34) to the constraint condition (25) leads to

$$
\int_{V} c^{\prime} d V+\int_{\Gamma} \dot{c}_{s} d S=\int_{\Gamma}\left(c+c_{s} \kappa\right) \mathbf{v} \cdot \mathbf{n} d S
$$

Making use of the above and (27) reduces $\dot{F}$ to

$$
\dot{F}=-\int_{\Gamma}\left\{\mathbf{n} \cdot\left[\llbracket \Sigma^{e}+(f-\bar{\mu} c) \mathbf{I} \rrbracket+\left(\gamma+f_{s}-\bar{\mu}_{s} c_{s}\right) \kappa \mathbf{I}\right]-\nabla_{s} \cdot\left(\boldsymbol{\sigma}^{s} \cdot \nabla_{s} \mathbf{u}\right)\right\} \cdot \mathbf{v} d S \quad .
$$

We further introduce the grand canonical potential of bulk and grand canonical potential of the interface as follows:

$$
\pi=f-\bar{\mu} c, \quad \pi_{s}=f_{s}-\bar{\mu}_{s} c_{s}
$$

Therefore, (40) becomes 


$$
\dot{F}=-\int_{\Gamma}\left\{\mathbf{n} \cdot\left[\llbracket \Sigma \rrbracket+\left(\gamma+\pi_{s}\right) \kappa \mathbf{I}\right]-\nabla_{s} \cdot\left(\boldsymbol{\sigma}^{s} \cdot \nabla_{s} \mathbf{u}\right)\right\} \cdot \mathbf{v} d S
$$

where $\Sigma=\Sigma^{e}+\pi \mathbf{I}=(w+\pi) \mathbf{I}-\boldsymbol{\sigma} \cdot \nabla \mathbf{u}$ denotes the generalized Eshelby's momentum tensor with the chemical free energy effect.

In order to guarantee the dissipation inequality condition, we choose

$$
\mathbf{v}=\mathbf{n} \cdot\left[\llbracket \Sigma \rrbracket+\left(\gamma+\pi_{s}\right) \kappa \mathbf{I}\right]-\nabla_{s} \cdot\left(\boldsymbol{\sigma}^{s} \cdot \nabla_{s} \mathbf{u}\right)
$$

so that

$$
\dot{F}=-\int_{\Gamma} \mathbf{v} \cdot \mathbf{v} d S<0
$$

As discussed earlier, the $\mathbf{v}=\mathbf{v}(\mathbf{x}, t)$ in (43) is the amount of displacement of the interface at the location $\mathbf{x}$ per unit change of $t$. In that sense, we may loosely call $\mathbf{v}$ the velocity of the interface. It should be noted, however, that this $\mathbf{v}$ generally does not represent the actual morphological evolution of the precipitate phase as the system approaches its chemo-mechanical equilibrium, which must be obtained by solving a non-equilibrium thermodynamic problem. Nevertheless, $\mathbf{v}$ represents a quasi-equilibrium process. Imagine that a binary system is given with an initial configuration of the precipitate phase, and a set of boundary conditions. If this system is in true chemo-mechanical equilibrium, then any configurational change of the precipitate phase will increase the system free energy. If this system is not in true chemo-mechanical equilibrium, diffusion will occur that leads to configurational change of the phases, or change of the precipitate/matrix interface. Such morphological evolution of the precipitate/matrix interface will continue until the system reaches its chemo-mechanical equilibrium. In theory, numerous paths can be taken to accomplish the evolution of the precipitate phase from its initial to the final chemo-mechanical equilibrium configuration. In a 
given problem, however, only of these paths is realized, which is dictated by the system parameters and the environment, including, for example, diffusivity of the solute. So, if one is interested in knowing the specific path to chemo-mechanical equilibrium, a full thermodynamic problem will need to be solved.

However, if one is only interested in knowing the final state of chemo-mechanical equilibrium, an alternative approach can be taken. As a thought experiment, we can consider the precipitate/matrix interface $\Gamma(t)$ as a function of the parameter $t$, a factitious time. So, the initial interface is $\Gamma(0)$. First, we hold this initial interface $\Gamma(0)$ in place, and let diffusion take place. After long enough time, diffusion will eventually cease, and the system becomes chemo-mechanically equilibrated for the fixed precipitate/matrix interface $\Gamma(0)$. Let $F(0)$ be the system free energy at this equilibrium state. Clearly, $F(0)$ can be calculated from (14) by using $\Gamma=\Gamma(0)$. At the next step $t$, we move the interface at $\mathbf{x}$ by an amount $\mathbf{v}(\mathbf{x}, t)$ according to (43) so the interface now becomes $\Gamma(t)$. Again, we now keep $\Gamma(t)$ fixed, and let the diffusion take place until the system reaches its chemo-mechanical equilibrium for the fixed precipitate/matrix interface $\Gamma(t)$. According to the foregoing derivations for $\mathbf{v}$, one must have $F(t)<F(0)$ if $t$ is small enough. The above steps can be repeated many times, each time with a small increment of $t$, then update the interface by $\mathbf{v}$ according to (43). As long as we hold the interface fixed at each update long enough so the system reaches its chemo-mechanical equilibrium, the system free energy at each stage of equilibrium will continue to decrease. It is then plausible that after enough number of increments, the system will arrive at it ultimate chemo-mechanical equilibrium, i.e., the $\mathbf{v}$ calculate from (43) will become zero (or negligibly 
small). This is the final state of chemo-mechanical equilibrium that we are seeking for. This thought experiment is in effect the numerical procedure that we use later to find the final state of chemo-mechanical equilibrium for given initial and boundary conditions.

It is also clear from the above descriptions, the parameter $t$ is not the real time, and the variable $\mathbf{v}$ is not the real speed of the interface. Eshelby (Eshelby, 1975) called the $\mathbf{v}$ configurational force, for it represents driving force acting on the interface to evolve the microstructure. The configurational force provides a convenient way to quantitatively understand the microstructural evolution of material defects, such as dislocations, cracks and inhomogeneities (Gurtin, 2000; Steinmann and Maugin, 2005).

To close this section, we state that, following directly from (43), the normal velocity of the interface is given by

$$
v_{n}=\mathbf{v} \cdot \mathbf{n}=\mathbf{n} \cdot \Sigma \cdot \mathbf{n}+\left(\gamma+\pi_{s}\right) \kappa-\mathbf{n} \cdot \nabla_{s} \cdot\left(\boldsymbol{\sigma}^{s} \cdot \nabla_{s} \mathbf{u}\right)
$$

\section{Numerical implementation}

The interface velocity in equation (45) is a function of chemical concentration, elastic deformation and interfacial energy/stress, which are determined by the group of equilibrium equations (26) - (28) and the mass conservation condition (25). These equations are fully coupled and need to be solved simultaneously. In this section, we will develop a hybrid nonlinear extended finite element method (XFEM) that combines with the level set method to compute the coupled chemo-mechanical fields. In the following development, unless noted otherwise, we will used boldface lowercase letters for vectors, boldface uppercase letters for matrices, and plain 
letters for scalar quantities. In this work, all the numerical implementations are carried out in the triangulated domain based on the finite element method.

we use the level set method (LSM) (Osher and Sethian, 1988) to implicitly describe the phase interface between the precipitate and the matrix. The interface $\Gamma$ is represented through the zero isocontour or the zero level set of a smooth function $\phi$ defined in the domain $V$, which can be stated as

$$
\Gamma=\{\mathbf{x}: \phi(\mathbf{x}, t)=0, \mathbf{x} \in V\},
$$

where $t$ is the same parameter introduced earlier. Further, for definiteness, the sign of $\phi$ can be chosen so that $\phi<0$ is in the precipitate and $\phi>0$ represents the matrix phase. Theoretically speaking, the level set function can be any smooth function defined in the domain. However, it is usually taken as the signed distance function in the numerical implementations. The level set evolution equation is given by

$$
\frac{\partial \phi}{\partial t}+v_{n}^{\phi}\|\nabla \phi\|=0 \quad \text { for } \quad \mathbf{x} \in V
$$

where $v_{n}^{\phi}$ is the normal velocity field associated with the level set function $\phi(\mathbf{x}, t)$.

Furthermore, the unit normal vector and the total curvature can be easily expressed in terms of level set function $\phi(\mathbf{x}, t)$, which are written as $\mathbf{n}^{\phi}=\nabla \phi /\|\nabla \phi\|, \kappa^{\phi}=-\nabla_{s} \cdot \mathbf{n}^{\phi}$. It can be easily shown that the unit normal vector $\mathbf{n}$ and the total curvature $\kappa$ of the interface $\Gamma$ defined by (46) is given by $\mathbf{n}=\left.\mathbf{n}^{\phi}\right|_{\mathbf{x} \in \Gamma}$ and $\kappa=\left.\kappa^{\phi}\right|_{\mathbf{x} \in \Gamma}$. In other words, $\mathbf{n}^{\phi}$ and $\kappa^{\phi}$ are extension values of $\mathbf{n}$ and $\kappa$ into $V$. 


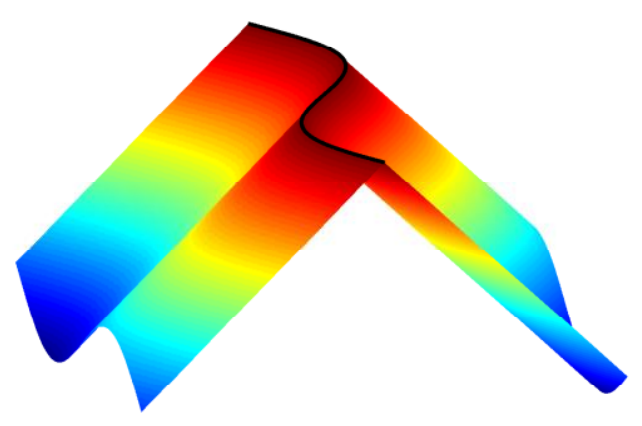

(a)

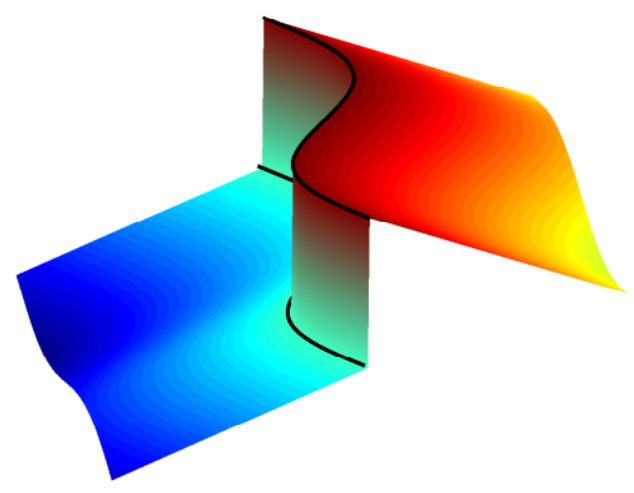

(b)

Fig. 1 Examples for functions with (a) weak discontinuity and (b) strong discontinuity.

The finite element equations will be based on the weak form of the chemo-mechanical equilibrium (24). Owing to the coherent interface assumption, the displacement $\mathbf{u}$ is continuous across the interface but its derivatives are not, which is known as 'weak discontinuity'. On the other hand, both the solution concentration $c$ and its gradient can be discontinuous across the interface, which is called 'strong discontinuity'. A comparison between the weakly discontinuous function and strongly discontinuous function is shown in Fig. 1. Because of their different types of discontinuities, different discretization schemes are needed for the displacement and the solute concentration fields, respectively,

$$
\mathbf{u}^{h}(\mathbf{x})=\mathbf{N}^{E}(\mathbf{x}) \mathbf{u}+\mathbf{F}^{E} \mathbf{N}^{E}(\mathbf{x}) \mathbf{a}, \quad c^{h}(\mathbf{x})=\mathbf{N}^{C}(\mathbf{x}) \mathbf{c}+\mathbf{F}^{C} \mathbf{N}^{C}(\mathbf{x}) \mathbf{d}
$$

where $\mathbf{N}^{E}$ and $\mathbf{N}^{C}$ are matrices of standard shape functions and $\mathbf{F}^{E}$ and $\mathbf{F}^{C}$ are matrices of enrichment functions introducing weak and strong discontinuities for the elastic and chemical fields, respectively. Here we use a corrected XFEM enrichment function for the displacement 
approximation to eliminating the side effects of blending elements (Fries, 2008), which gives

$$
F_{i}^{E}(\mathbf{x})=N_{i}(\mathbf{x})\left[|\phi(\mathbf{x})|-\left|\phi\left(\mathbf{x}_{i}\right)\right|\right] R(\mathbf{x}),
$$

where $\phi$ is the level set function and $R(\mathbf{x})$ is given by

$$
R(\mathbf{x})=\sum_{i \in I^{*}} N_{i}(\mathbf{x}),
$$

in which $I^{*}$ is the nodal set containing the enriched nodes. It is noted that $R(\mathbf{x})$ is equal to 1 in an enriched element, but forms a ramp function in a blending element.

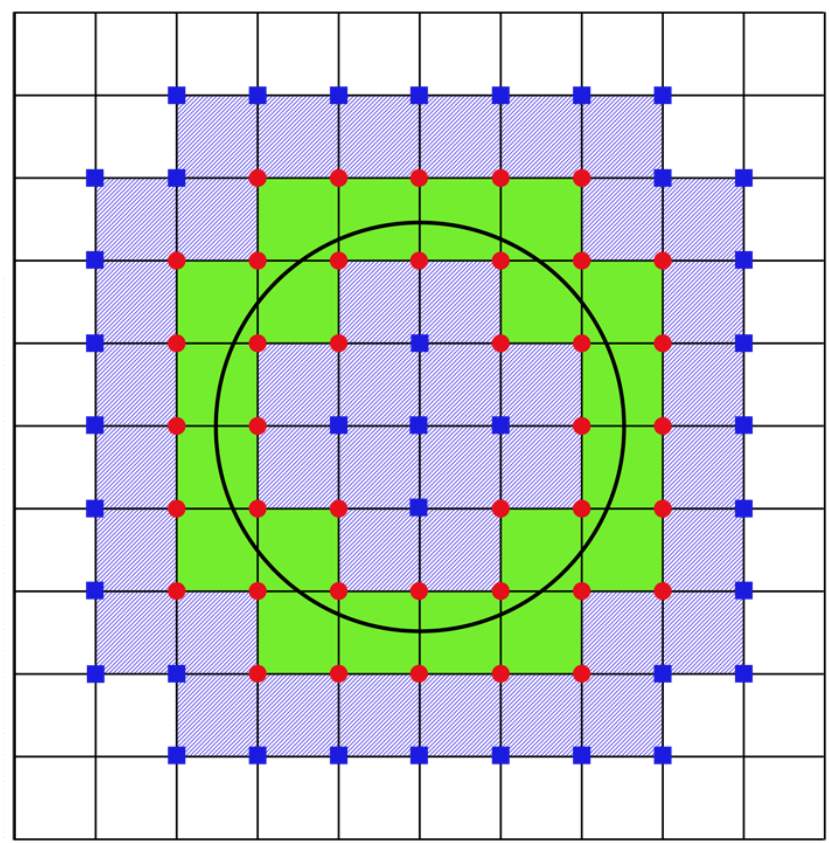

Standard elements

$\square$ Reproducing elements

Blending elements

- Enriched nodes

- Blending nodes

Fig. 2 The discretized domain with reproducing elements and blending elements and their enriched/blending nodes

Since the chemical field contains a strong discontinuity, the Heaviside step function is used.

Such enrichment scheme does not lead to problems in the blending elements for standard XFEM (Bordas et al., 2007; Fries, 2008). Thus we have

$$
F_{i}^{C}(\mathbf{x})=N_{i}(\mathbf{x})\left[H(\phi(\mathbf{x}))-H\left(\phi\left(\mathbf{x}_{i}\right)\right)\right],
$$

where 


$$
H(\phi)=\left\{\begin{array}{ll}
1, & \phi>0 \\
0, & \phi \leq 0
\end{array} .\right.
$$

It should be pointed out that the enrichment functions (49) and (51) are local functions near the interface and vanish in standard elements far away from the interface. In other words, the extra enriched degrees of freedom caused by the enrichments only exist in local elements near the interface, see Fig. 2. As we can see, the XFEM allows the geometric interface to pass through the elements so that the geometry of the phase interface can be independent of underlying mesh, and no remeshing is needed when interface moves (Belytschko et al., 2001). This provides great advantages for problems with moving interfaces.

With the above discretized element quantities, we can assemble the global FEM equations with coupled global matrix and load vectors in a standard way. Our coupled XFEM/LSM codes are built on top of a C++ finite element library FEniCS/dolfin (Logg and Wells, 2010), and linear triangular elements are used in the simulation. It is worth pointing out that due to the nonlinearity in the chemical potential in (7), the discretized system of algebraic equations are nonlinear. Therefore, an iterative solver, e.g. the Newton method, is needed to obtain the numerical solution. Due to the hyperbolic nature of the level set equation, a Galerkin least squares (GLS) finite element scheme (Chessa et al., 2002) is used to solve equation (47), and a first order forward Euler method is adopted for the time discretization. In addition, a fast marching method on the finite element grid is devised for constructing the extension velocities and the periodically re-initialize the level set function (Zabaras et al., 2006). For more detailed discussions on level set method and fast marching method, the readers are referred to references (Osher and Fedkiw, 
2002; Sethian, 1996).

\section{Results and discussion}

In this section, we limit studies to a two-dimensional plain-strain deformation. For simplicity, we make the following assumptions in the reminder of this paper:

- The bulk elastic constants are independent of concentration so that $\frac{\partial \mathbf{S}}{\partial c}=0$.

- The bulk eigenstrain of is isotropic, i.e., $\boldsymbol{\eta}=\eta \mathbf{I}$.

- In the bulk chemical free energy of (8), $B_{m, p}=0$ and $A_{m, \mathrm{p}}=f_{0}$.

- The interfacial properties are independent of the solute concentration, i.e., $\boldsymbol{\eta}^{s}=\mathbf{0}$ and $f_{s}\left(c_{s}\right)=0$. In the interfacial elastic excess energy, $\boldsymbol{\tau}^{s}=\mathbf{0}$ and $\mathbf{C}^{s}=\mathbf{0}$ so that $\gamma=\gamma_{0}=$ costant

- All the calculations are performed using the elastic constants of $\gamma-\gamma^{\prime}$ Ni-alloys with cubic anisotropy, $C_{11}^{P}=179 \mathrm{GPa}, C_{12}^{P}=123 \mathrm{GPa}$ and $C_{44}^{P}=81 \mathrm{GPa}$ for the precipitate phase, and $C_{11}^{M}$ $=161 \mathrm{GPa}, C_{12}^{M}=107 \mathrm{GPa}$ and $C_{44}^{M}=85 \mathrm{GPa}$ for the matrix (Fahrmann et al., 1999).

For convenience, we introduce the dimensionless solute concentration,

$$
\bar{c}=\frac{c-c_{m}}{c_{p}-c_{m}}
$$

This leads to

$$
\bar{c}=\left\{\begin{array}{ll}
0 & \text { when } c=c_{m} \\
1 & \text { when } c=c_{p}
\end{array}, \quad 1-\bar{c}=-\frac{c-c_{p}}{c_{p}-c_{m}} .\right.
$$

Consequently, Eq. (7) can be simplified to $f(c)=f_{0}\left(c_{p}-c_{m}\right)^{2} \bar{f}(\bar{c})$, where 
$\bar{f}=\min \left\{\bar{c}^{2},(1-\bar{c})^{2}\right\}$. The term $f_{0}\left(c_{p}-c_{m}\right)^{2}$ can be viewed as the magnitude of the bulk chemical free energy. Similarly, the compositional strain of can be written as $\boldsymbol{\varepsilon}^{c}=\varepsilon_{0} \overline{\boldsymbol{\varepsilon}}^{c}$, where $\varepsilon_{0}=\eta\left(c_{p}-c_{m}\right), \overline{\boldsymbol{\varepsilon}}^{c}=\left(\bar{c}-\bar{c}_{r}\right) \mathbf{I}$, and $\bar{c}_{r}=\left(c_{r}-c_{m}\right) /\left(c_{p}-c_{m}\right)$. Clearly, the dimensionless parameter $\varepsilon_{0}$ gives a measure of the coupling between the chemical and mechanical fields. In the absence of such coupling, the elastic field vanishes since no other externally applied loads are present. Analogously, the strain energy density can be recast into $w=w_{0} \bar{w}$, where $w_{0}=C_{44}^{p} \varepsilon_{0}^{2}$, $\bar{w}=(1 / 2)\left(\overline{\boldsymbol{\varepsilon}}-\overline{\boldsymbol{\varepsilon}}^{c}\right): \overline{\mathbf{C}}:\left(\overline{\boldsymbol{\varepsilon}}-\overline{\boldsymbol{\varepsilon}}^{c}\right), \quad \overline{\mathbf{C}}=\mathbf{C} / C_{44}^{p}$ and $\overline{\boldsymbol{\varepsilon}}=\boldsymbol{\varepsilon} / \varepsilon_{0}$ The parameter $w_{0}$ can then be considered as the magnitude of the elastic strain energy. By making use of the above, the system's total free energy function can be written as $F=\gamma_{0} R_{0}^{2} \bar{F}$ where the dimensionless system free energy is given by

$$
\bar{F}=\beta \int_{\bar{V}}(\alpha \bar{f}+\bar{w}) d \bar{V}+\int_{\bar{\Gamma}} d \bar{S}
$$

where

$$
\alpha=\frac{f_{0}\left(c_{p}-c_{m}\right)^{2}}{w_{0}}=\frac{f_{0}}{C_{44}^{p} \eta^{2}}, \quad \beta=\frac{w_{0} R_{0}}{\gamma_{0}}=\frac{C_{44}^{p} \varepsilon_{0}^{2} R_{0}}{\gamma_{0}}
$$

In the above, the parameter $R_{0}$ is a characteristic length of the precipitates given by $R_{0}=\left(3 V_{0} / 4 \pi\right)^{1 / 3}$ in three-dimensions and $R_{0}=\left(A_{0} / \pi\right)^{1 / 2}$ in two-dimensions, where $V_{0}$ and $A_{0}$ are the initial volume and area of the largest precipitate. The coordinates are also normalized by $\bar{x}_{i}=x_{i} / R_{0}$, which transforms $V$ to $\bar{V}$ and $\Gamma$ to $\bar{\Gamma}$. The dimensionless parameter $\alpha$ represents the ratio of chemical energy density and elastic strain energy density. Larger $\alpha$ means the chemical energy is dominant over the elastic strain energy, and vice versa. The dimensionless parameter $\beta$ gives the ratio of the bulk elastic strain energy density and the 
interfacial energy density. Large $\beta$ means the bulk elastic strain energy is dominant over the interfacial energy, and vice versa. Therefore, for a fixed $\alpha$, increasing $\beta$ means decreasing surface energy while keeping chemical and elastic energies the same. Alternatively, for a fixed $\alpha$, increasing $\beta$ means increasing both elastic and chemical energies (so their ratio stays the same since $\alpha$ is fixed) while keeping the interfacial energy the same.

The numerical results presented below are all for two-dimensional plane strain. In addition, the Cartesian axes are chosen to coincide with the cubic crystallographic orientations. The reference concentration $\bar{c}_{r}$ is set equal to the average value of the entire system. In the numerical examples, we use $\bar{c}_{r}=\bar{c}_{0}=0.1$, which implies that the total volume fraction of precipitates under equilibrium should be $10 \%$ in the absence of elastic strain energy and interfacial energy.

\subsection{A single precipitate}

We first consider the equilibrium shapes of a single misfit particle embedded in an elastic media. In experimental investigations, the parameter $\beta$ can be changed by adjusting the value of the misfit strain. It has been shown that as the magnitude of misfit increases ( $\beta$ increases), the particle shapes become progressively less spherical and more cube-like in nature. In addition, the particle size also increases with increasing misfit strains (Fahrmann et al., 1995), which is considered a result of the competition between the bulk and interfacial energies. In this numerical example, we consider a misfit particle with a circular initial shape and radius $R_{0}$. The simulation domain size is taken as $10 \times 10 R_{0}^{2}$.

We first consider the case where the ratio between the bulk chemical and elastic energy 
densities is fixed at $\alpha=10$. For different ratios between the elastic bulk and interfacial energy densities, $\beta=2,5,10,20$, the corresponding equilibrium configurations of an isolated single

particle are shown in Fig. 3. Two observations can be made based on these figures. First, lower interfacial energy (larger $\beta$ ) leads to square-like shape with sharper corners and flatter sides, while higher interfacial energy (smaller $\beta$ ) leads to circle-like shape with smooth corners and curved sides. Second, higher interfacial energy (smaller $\beta$ ) favors smaller particle size. These observations can be explained by the system's tendency to reduce its total free energy. When $\beta$ is large, the interfacial energy is unimportant, and the bulk chemical and elastic strain energies dominate. Due to cubic symmetry of elasticity, the particle takes square-like shape to minimize its elastic strain energy. When $\beta$ is small, the interfacial energy dominates. Since the interfacial energy is assumed isotropic, its tendency is to minimize the particle size and to make the shape circular/spherical to minimize the interfacial perimeter/area. Those results show the same qualitative trend as the experimental observations mentioned above (Fahrmann et al., 1995).

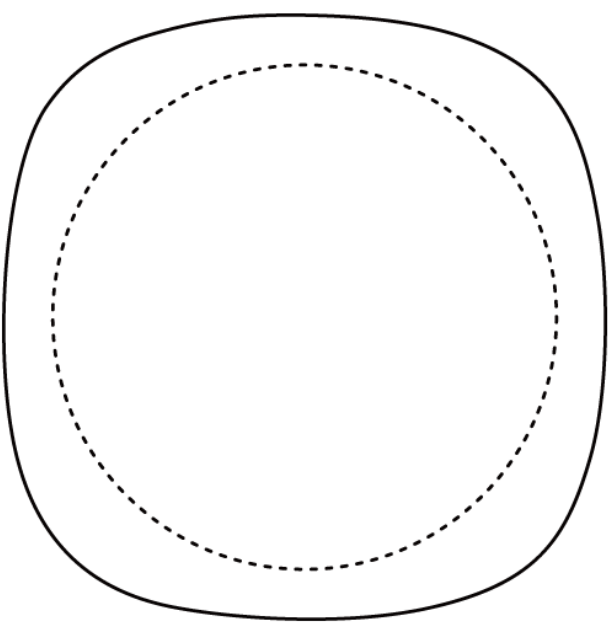

(a)

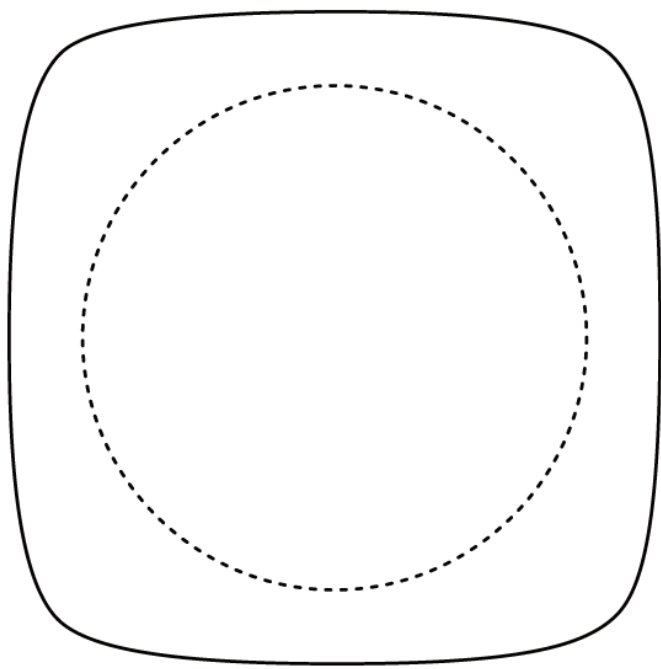

(b) 


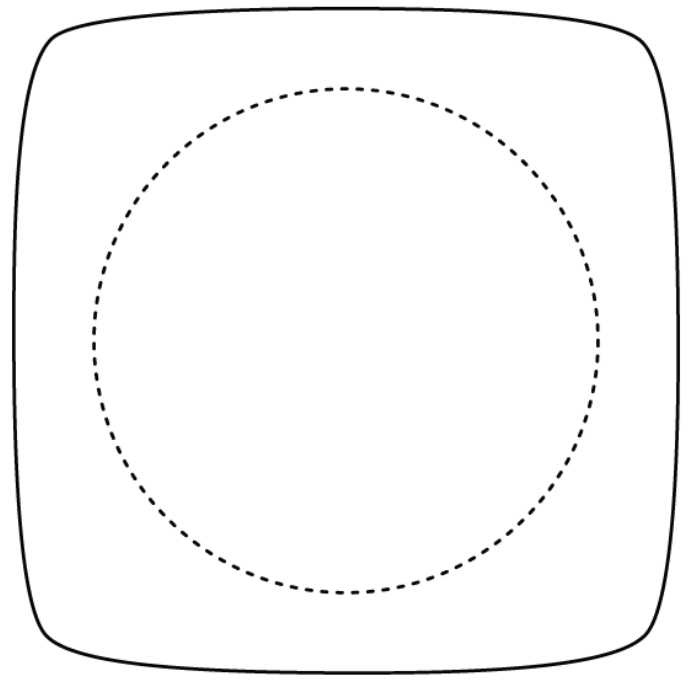

(c)

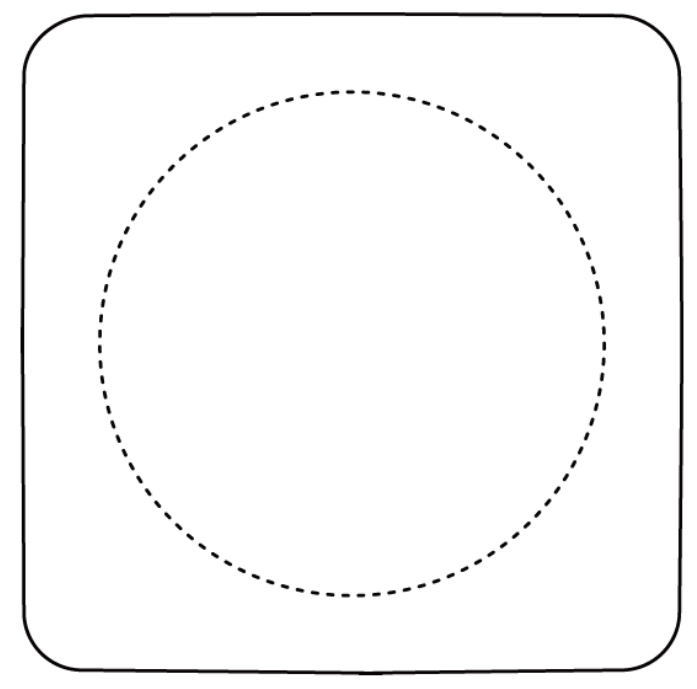

(d)

Fig. 3 Equilibrium shape (solid line) of a single particle in a large matrix with $\alpha=10$ and (a) $\beta=2$, (b) $\beta=5$, (c) $\beta=10$, and (d) $\beta=20$. The dotted line represents the initial shape of the particle.

Fig. 4 shows the variation of system energies with respect to the (fictitious) time steps for particles with different initial $\beta$. The energies are all normalized by their total energy values at their initial states. It is not surprising that with increasing time, the total system energy decreases and gradually converges to a constant value as the system approaches its equilibrium state. The results show that the interfacial energy is a significant part of the total system energy for $\beta=2$, while for $\beta=20$ the interfacial energy is almost negligible, and the chemical and elastic energy become more prominent. In addition, we also notice that both elastic energy and interfacial energy increase during the particle growth. However, their increase is more than compensated by the decrease of the chemical energy so that the total system energy still decreases. One may then 
conclude that the overall kinetic process of particle growth is driven or controlled by the chemical energy. To verify this conclusion, we note that the relative magnitude between the chemical and elastic energies is characterized by the parameter $\alpha$. The results shown so far are for $\alpha=10$. If we reduce $\alpha$, one would expect that the overall evolution kinetics will be controlled by the elastic energy.

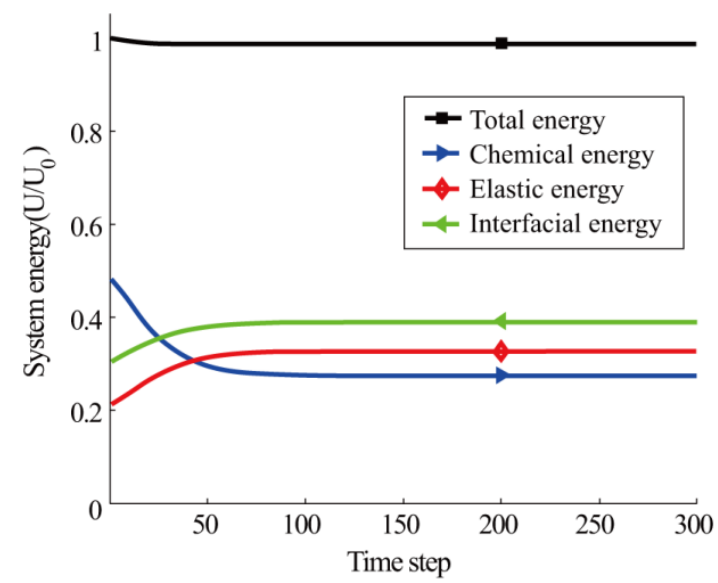

(a)

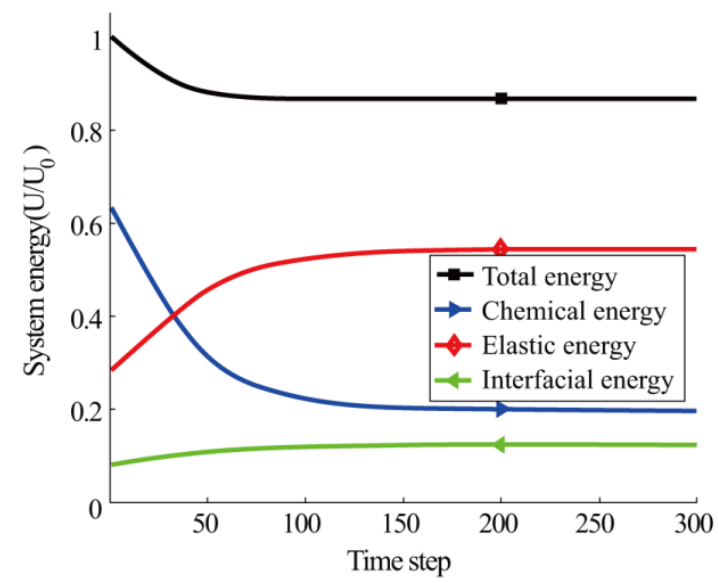

(c)

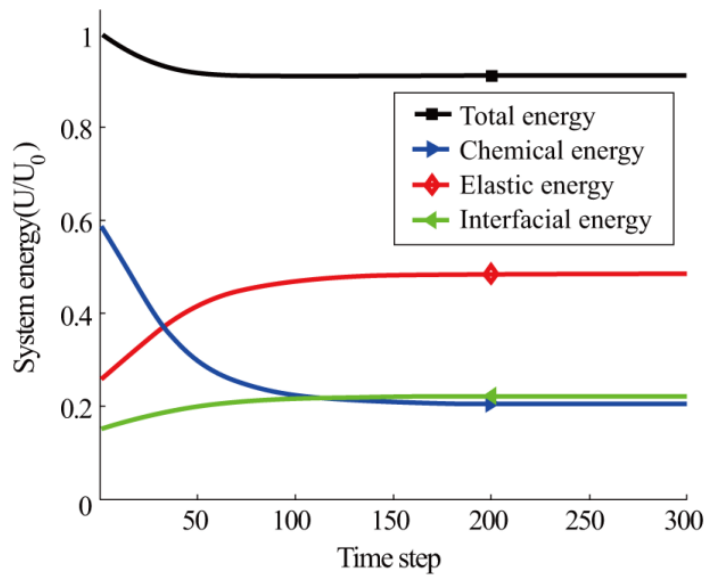

(b)

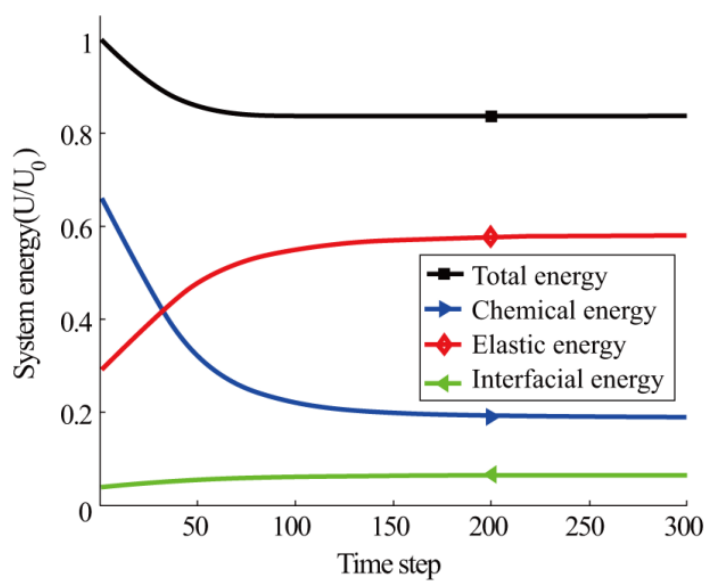

(d)

Fig. 4 Variation of normalized energies vs. time steps for the system with a single particle in a 
large matrix with $\alpha=10$ and (a) $\beta=2$, (b) $\beta=5$, (c) $\beta=10$, and (d) $\beta=20$.

To this end, we repeated the above calculations with $\alpha=5$. Fig. 5 shows the corresponding equilibrium shapes for a single particle in a large matrix with $\beta=10$ and $\beta=20$, respectively. It is clearly seen that the particle at equilibrium has flatter edges and larger size when $\beta=20$, which is similar to the previous example. However, they obviously have smaller particle size compared to their counterparts in the case of $\alpha=10$. We note that particles with initial $\beta=2$ and $\beta=5$ tend to shrink and finally disappear during the evolution. This can be also observed from the energy evolution curve in Fig. 6, where we can see from Fig. 6(a) and (b) that both elastic energy and interfacial energy decrease and gradually reduce to zero when the misfit particle disappears. In these two cases, the particle cannot stably exist due to the combined effects of elastic energy and interfacial energy, even if particle nucleation occurs. Therefore, we can remark that both the interfacial energy and the elastic energy can significantly suppress the nucleation and growth of particles.

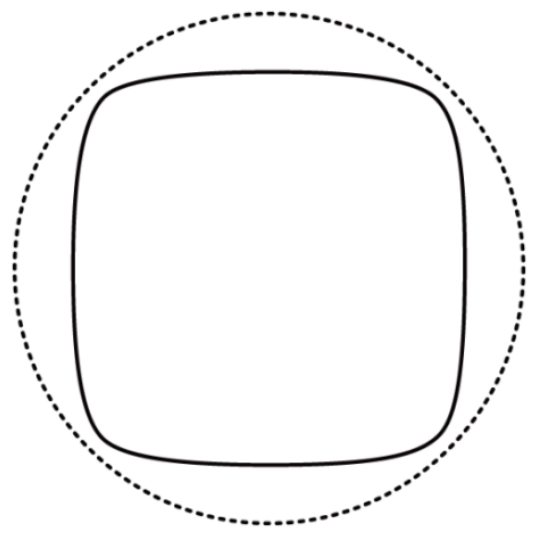

(a)

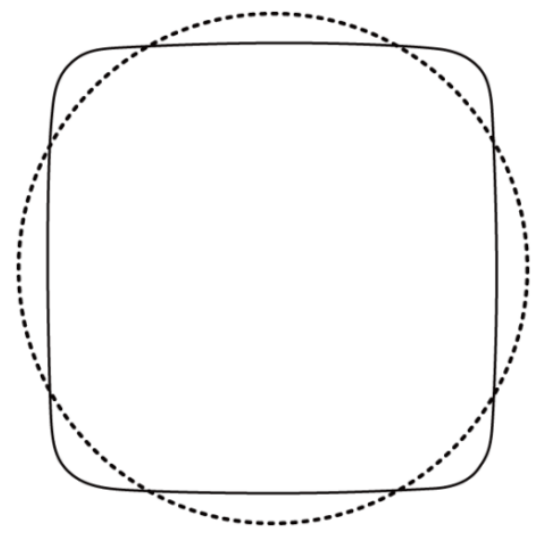

(b) 
Fig. 5 Equilibrium shape (solid line) of a single particle in a large matrix with $\alpha=10$ and (a) $\beta=10$, and (b) $\beta=20$. The dotted line represents the initial shape of the particle. Particles with initial $\beta=2$ and $\beta=5$ shrink and finally disappear during the evolution.

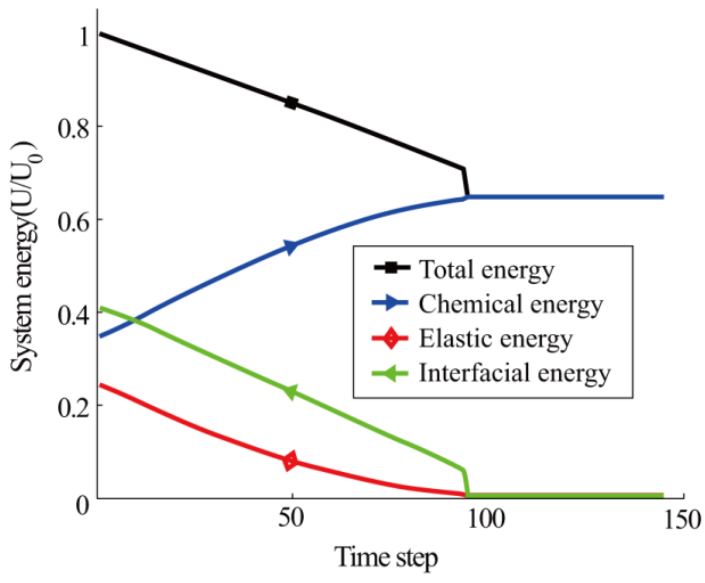

(a)

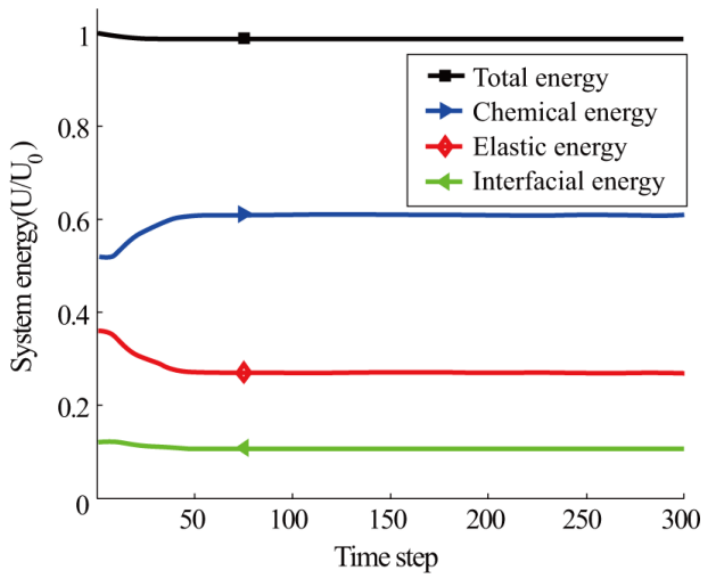

(c)

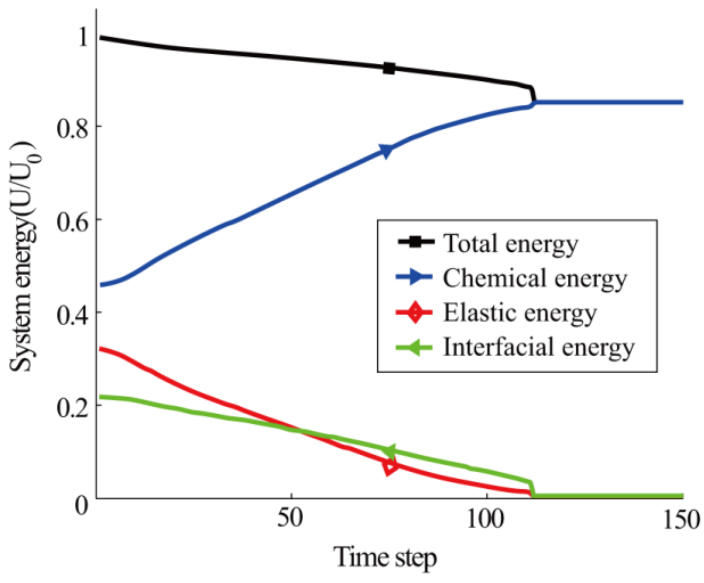

(b)

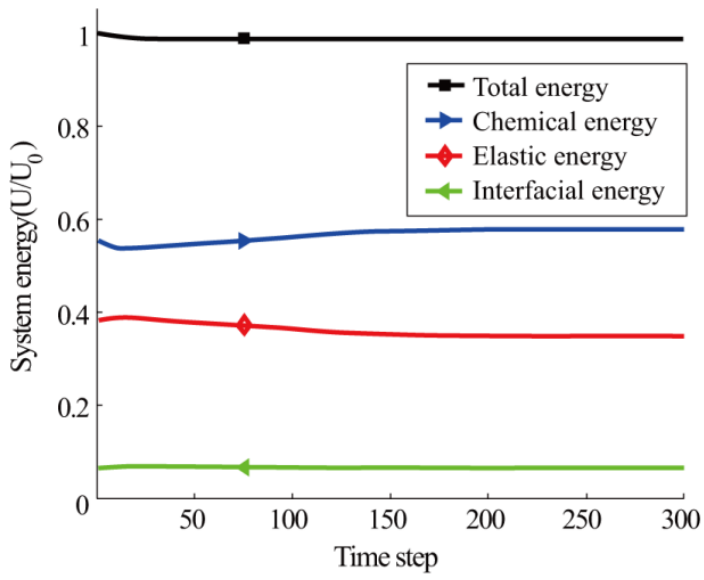

(d)

Fig. 6 Variation of normalized energies vs. time steps for the system with a single particle 
in a large matrix with $\alpha=5$ and (a) $\beta=2$, (b) $\beta=5$, (c) $\beta=10$, and (d) $\beta=20$.

These foregoing results show a collaborative competition among three driving forces, the bulk elastic strain energy, the interfacial energy and the bulk chemical energy. The final equilibrium state of the system is a compromise among these three energies in their individual pursuit to minimize themselves so that the total system energy is a minimum under the given initial condition. Since the initial average solute concentration is super-saturated, the chemical energy drives the solute to separate into matrix and precipitate phases. On the other hand, phase separation increases the bulk elastic strain energy. So, the elastic strain energy works against phase separation. In a similar manner, the interfacial energy dislikes interfaces. So, it works together with the elastic energy to suppress phase separation by minimizing the interfacial area. However, once a particle is formed, interfacial energy turns against the bulk elastic strain energy by making the particle as circular as possible, while the bulk elastic energy tries to make the particle as square as possible due to cubic anisotropy. Overall, if the bulk chemical energy dominates (e.g., $\alpha=10$ ), it is the reduction of chemical energy that drives the overall evolution. Consequently, the particle grows in size to reduce the chemical energy, even though in doing so the bulk and interfacial elastic energies both have to increase. If the bulk elastic strain energy dominates (e.g., $\alpha=5$ ), it is the reduction of bulk elastic strain energy that drives the overall evolution. Consequently, the particle shrinks in size to reduce the elastic strain energy, even though in doing so the bulk chemical energy has to increase. In fact, with the help of higher $(\beta \leq 5)$ interfacial energy, phase separation is completed suppressed for the case of $\alpha=5$. 
Another interesting aspect is the actual distribution of solutes in the different phases. In the absence of the bulk and interfacial elastic energies, the solute concentration in each phase is uniform. In the presence of the bulk and interfacial elastic energies, we expect that the concentration distribution be influenced by the elastic deformation. Fig. 7 shows the normalized concentration profiles along different crystallographic directions through the particle center at the equilibrium state. The solid and dashed lines represent the distributions along $\langle 100\rangle$ and $<110>$ directions, respectively. It is obvious that the concentrations are not uniform in either phases, especially when the elastic energy is large ( $\alpha$ is small). Also, the values of the concentration in both phases are slightly deviated from their theoretical values in the absence of the bulk and interfacial elastic energies.

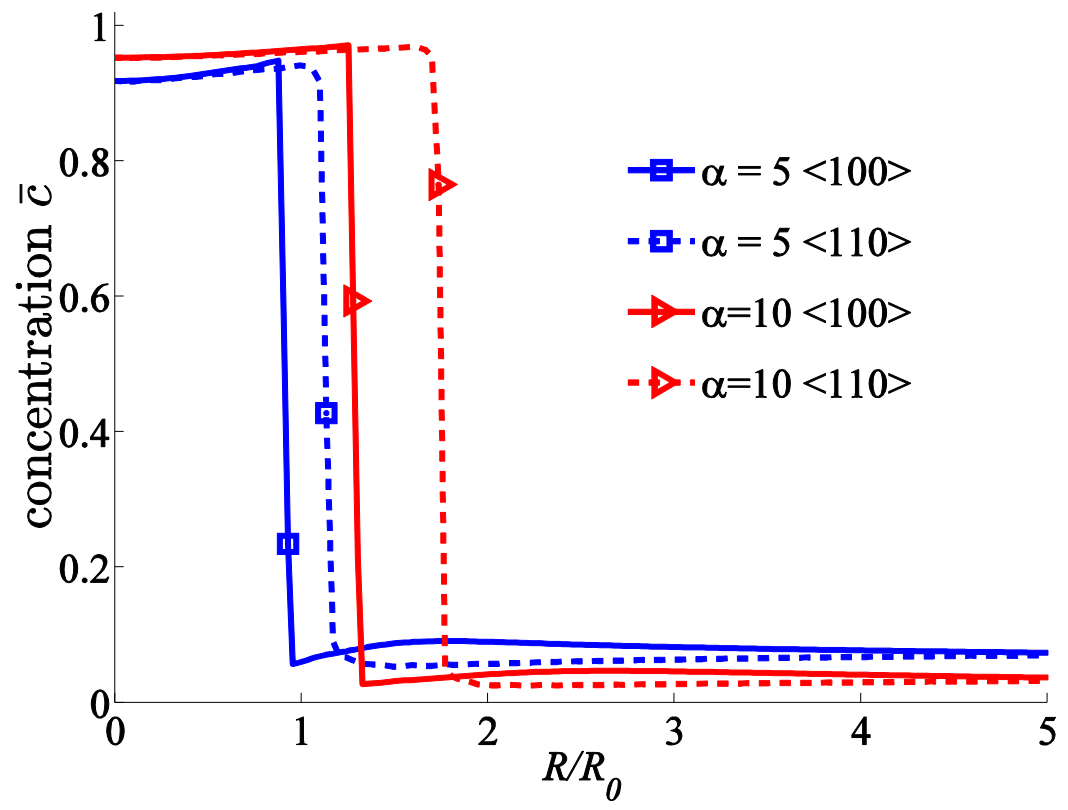

Fig. 7 Equilibrium concentration profiles along different crystallographic directions when $\beta=20$ 


\subsection{Double particles}

We now consider the evolution and equilibrium morphologies of two interactive particles. It is experimentally observed that, during thermal aging, the precipitate structure proceeds from one that is initially with a large number of small particles to one in which the cubic precipitates become more and more aligned along [100] directions. Upon further aging the particles either coalesce or coarsen into plates (Ardell and Nickolson, 1966; Ma and Ardell, 2007). The number of precipitates in $\mathrm{Ni}-\mathrm{Al}$ alloys decreases significantly and the surviving precipitates are mostly slim rod-like plates aligned with [100] directions.

The two-particle system has been studied previously by researchers using the boundary integral equation methods (Leo et al., 2000; Su and Voorhees, 1996b). It has been shown that particles in $\mathrm{Ni}-\mathrm{Al}$ alloys attract each other and tend to merge into one particle. However, the merging and post-merging phenomena were not simulated in those works due to the limitations of the numerical techniques used in their simulations. In this section, we focus on two equal-sized particles with circular initial shapes and radius $R_{0}$. We define the angle between the line connecting the centers of these two particles and the [100] direction as $\theta$. The simulation domain size is set as $13 \times 10 R_{0}^{2}$.

First, we consider two particles with a center-to-center distance of $3 R_{0}$. They are initially aligned along an [100] direction, i.e. $\theta=0$. Shown in Fig. 8 are the final equilibrium shapes (solid lines) of these two particles for $\alpha=10$ and different values of $\beta$. The dotted lines represent the initial configurations for the two-particle system. When $\beta$ is large $(\beta \geq 5)$, the elastic strain energy density is (relatively) large in comparison with the interface energy density. 
So the system evolves predominantly by the tendency to reduce the elastic strain energy.

Consequently, the two particles merge into one elongated particle along a [100] direction due to elastic anisotropy. The larger the $\beta$, the more elongated the particle is. Although a more elongated particle has large interface area which increases the total surface energy, the decrease of the elastic and chemical energies is still more than the increase of the interfacial energy, which makes the rod-like morphologies more favorable. These results are consistent with the experimental observations (Ardell and Nickolson, 1966; Ma and Ardell, 2007) that, during thermal aging, the number of precipitates in Ni-Al alloys decreases significantly and the surviving precipitates are mostly slim rod-like particles aligned with [100] directions.

For smaller $\beta$, for example, $\beta=2$, the particles move towards each other slightly, but do not coalesce. This seems to be counter intuitive, since smaller $\beta$ means that the interfacial energy density is (relatively) large in comparison with the elastic strain energy density, which should favor particle merging in order to reduce the interface surface area. However, high interfacial energy density also prevents the particle from growing in volume. So, the only way the particles can merge is to move towards each other. However, simply moving the particles toward each other without changing their volumes does not change the bulk chemical and elastic strain energy, which means that these is very little driving force for doing so. Therefore, the particles in this case ( $\beta=2)$ remain more or less their initial volumes and locations. For large $\beta$, the interfacial energy is unimportant so that the particle is able to grow in volume to reduce the chemical energy, which leads to their coalescence. These simulation results are in agreement with the results obtained from the boundary integral methods (Jou et al., 1997; Su and Voorhees, 
1996b).

For the case of $\alpha=5$, our numerical simulations show that the two-particle system behave similarly as the case of $\alpha=10$, except that particles with small $\beta$ disappear because the (relative) high interfacial energy suppresses the nucleation of precipitates, which occurs in single particle cases as well.

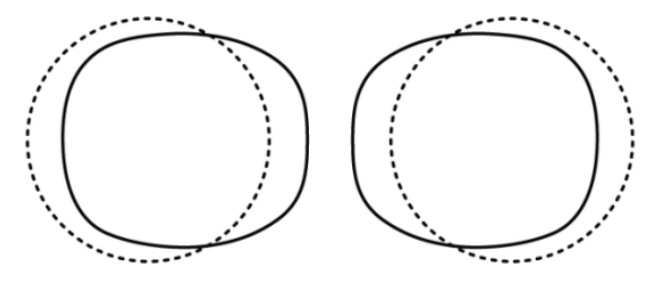

(a)

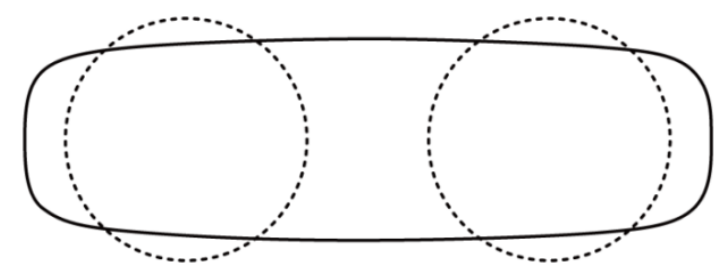

(b)

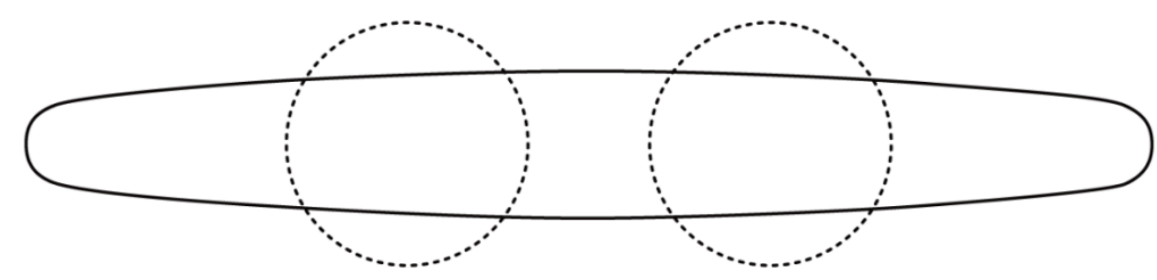

(c)

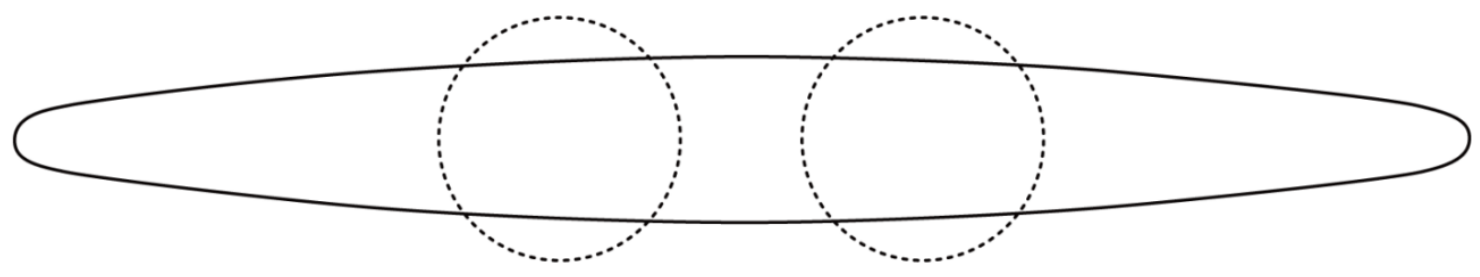


Fig. 8 Equilibrium shapes (solid lines) of two-particle systems in a large matrix with $\alpha=10$ and (a) $\beta=2$, (b) $\beta=5$, (c) $\beta=10$, and (d) $\beta=20$. The dotted lines represent the initial shapes of particles.

Next, we consider another case where two equal-sized particles are initially aligned in a different crystallographic direction, for example, $\theta=30^{\circ}$. Fig. 9 shows the initial and final equilibrium shapes of two particles under different initial conditions. Again, the dotted lines and the solid lines represent the initial configurations and finial equilibrium configurations of the two-particle system, respectively. It is interesting to see that the same final equilibrium morphologies as those in previous examples are observed, although particles are initially placed in completely different crystallographic directions. In all the cases we considered here, the particles first re-adjust their positions to align with an [100] direction, which is elastically soft, in order to reduce the elastic energy. Afterwards, the particles coalesce into one and further elongate along the [100] direction.
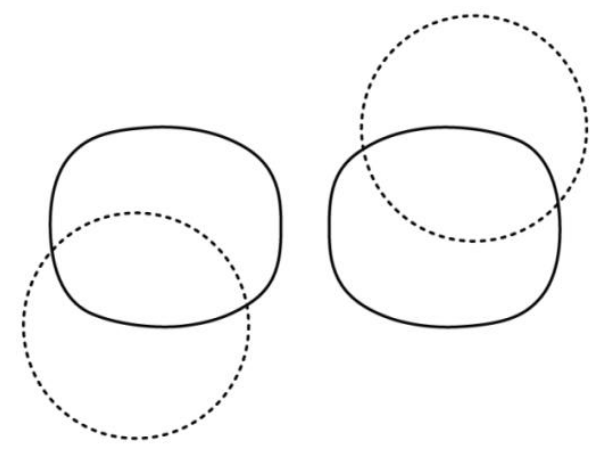

(a) 


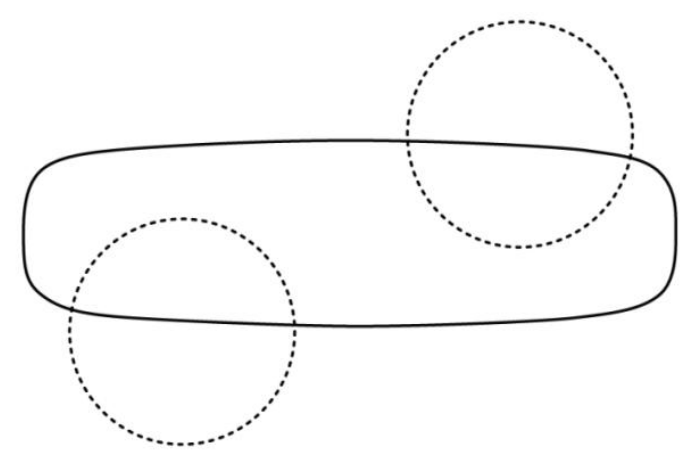

(b)

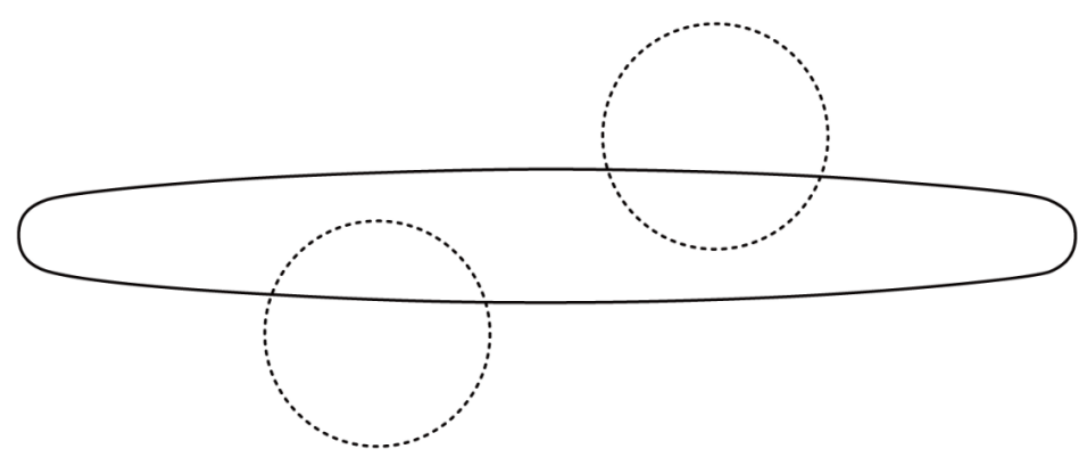

(c)

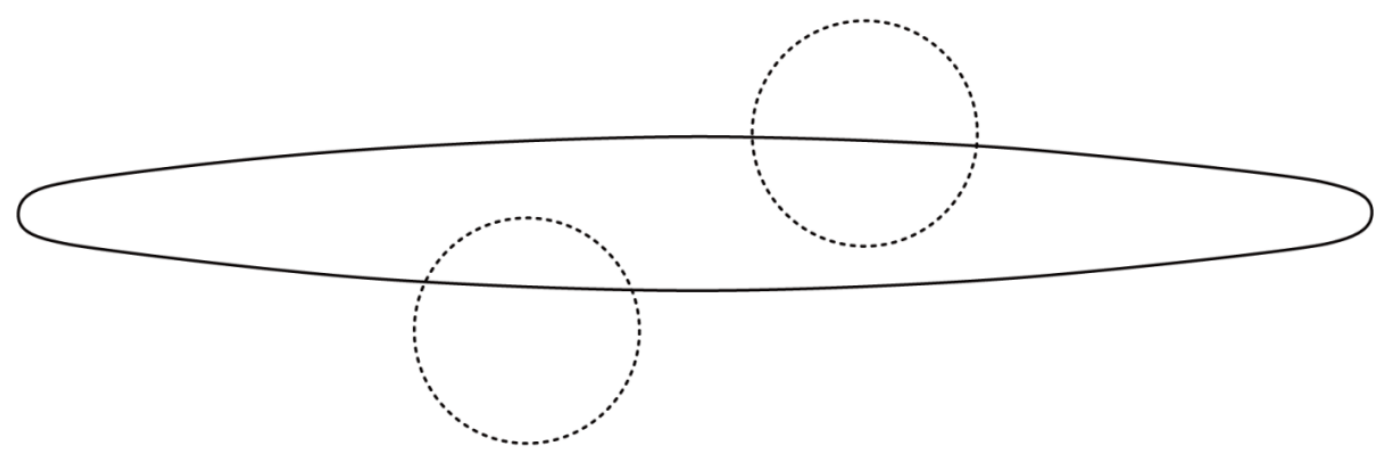

(d)

Fig. 9 Equilibrium shapes (solid lines) of two particle in a large matrix with $\alpha=10$ and (a) $\beta=2$, (b) $\beta=5$, (c) $\beta=10$, and (d) $\beta=20$. The dotted line represents the initial shape of the particle.

For better visualization, we show two typical evolution processes for the two-particle system 
with different initial configurations $\theta=0$ in Fig. 10(a) and $\theta=30^{\circ}$ in Fig. 10(b), respectively. The arrow the figures indicates the direction of increasing $t$. It is seen that the particles change their sizes, shapes, as well as positions, and finally merge. After merging, the merged single particle keeps growing along a [100] direction.

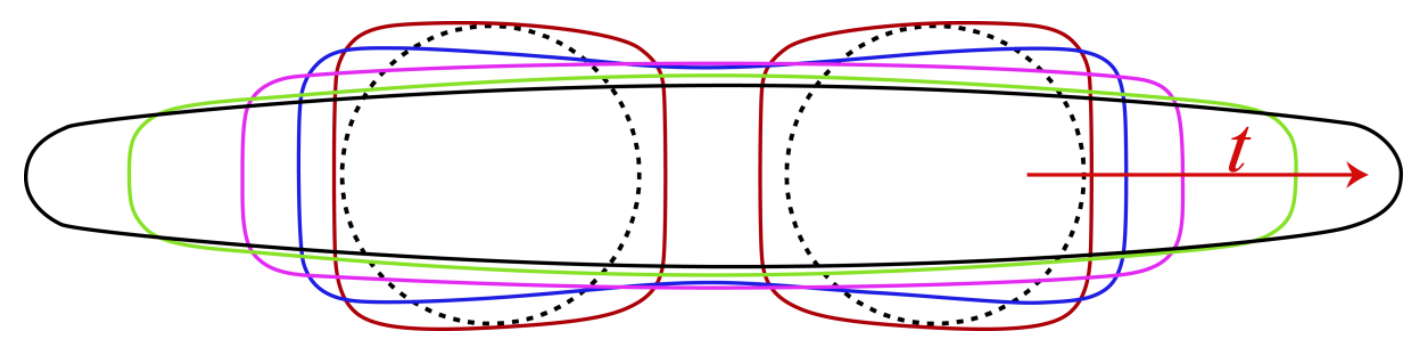

(a)

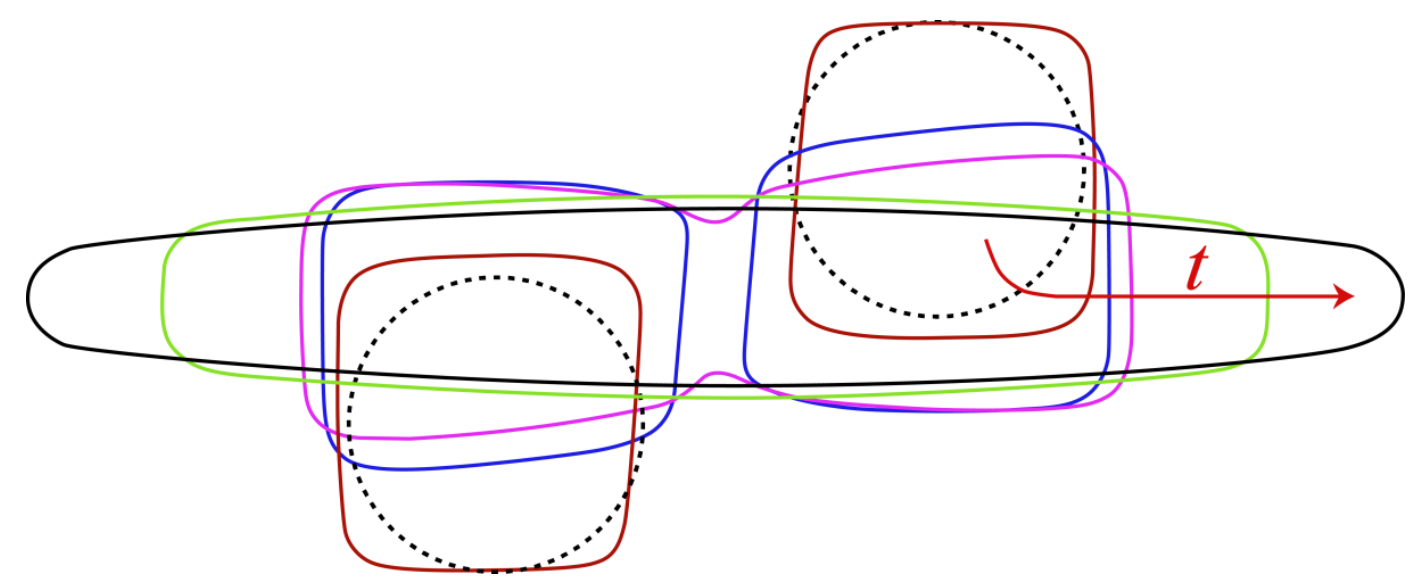

(b)

Fig. 10 Morphological evolution of two-particle systems with dilatational eigenstrain when $\beta=10$ (a) $\theta=0$, (b) $\theta=30^{\circ}$. The dotted lines represent the initial shapes of particles and the arrows point to the evolution directions of interfaces when time step $t$ increases. 


\section{Conclusions}

This paper focused on the effects of elastic and interfacial energies on equilibrium morphologies of misfit particles due to phase separation in binary alloys under chemo-mechanical equilibrium conditions. First, a continuum framework that governs the chemo-mechanical equilibriums of both the bulk and interface is developed using a variational approach by treating the phase interface as a sharp interface endowed with interfacial excess energy. The evolution of the interface is described by a level set method. The evolution kinetics of the interface is also derived and is related to the level set evolution equation. Further, an explicit expression of the configurational force that drives the morphological evolution of the phases are derived, which contains the generalized Eshelby's momentum tensor and the chemical and interfacial effects. Second, an extended finite element method (XFEM) in conjunction with the level set method is developed to simulate the behavior of the coupled chemo-mechanical system. The nonlinear XFEM is capable of solving the coupled chemical and elastic fields with sharp interfaces without remeshing when the phase interface evolves.

By using the XFEM developed here, numerical examples were carried out on $\mathrm{Ni}$-Al alloys to investigate the effects of chemical energy, elastic strain energy, and the interfacial energy on the morphological evolution of particles in different material systems. The simulated results show that the morphological evolution of a phase separated binary alloy is governed by the competition among three energetic driving forces, bulk chemical energy, bulk elastic strain energy and interfacial excess energy. To study the roles that these energies play, two dimensionless parameter $\alpha$ and $\beta$ are introduced, which represent the ratio between the bulk 
chemical free energy density and the bulk elastic strain energy density, and the ratio between the bulk elastic strain energy and the interfacial excess energy, respectively. Under a given $\alpha$, in material systems with larger $\beta$, the interfacial excess energy is relatively low. Therefore, misfit particles tend to grow in size and may eventually coalesce. The negligible interfacial excess energy density (which is assumed isotropic) also leads to square-like or rod-like precipitate shapes due to the system's tendency to reduce its elastic strain energy by growing the misfit particle along elastically soft directions. On the contrary, for systems with smaller $\beta$, interfacial excess energy dominates. Thus, misfit particles are usually spherical, smaller ones tend to shrink in size and may eventually disappear. Multiple misfit particles may choose to stay separated because the high interface energy suppresses particle growth, and there is very little driving force for the particle to move toward each other without changing their volumes. In general, misfit particles tend to align themselves along an elastically soft direction. Under given $\beta$, material systems with larger $\alpha$ have higher chemical energy, and lower elastic strain energy, which favors separation. Thus, misfit particles tend to grow. On the other hand, the same misfit particles in systems with smaller $\alpha$ may shrink and eventually disappear.

The simulated behavior for the Ni-alloys agrees well with experimental observations, demonstrating that the coupled chemo-mechanics model together with the numerical techniques developed here provides an efficient simulation framework to predict the equilibrium morphologies of precipitates in phase separate alloys. However, we would like to restate that the formulation of the evolution dynamics in this paper is based on the assumption of chemo-mechanical equilibria. For the real time-dependent microstructural evolution, 
non-equilibrium thermodynamics with transient mass diffusion is required, which will be presented in a separate publication. In addition, the numerical results presented here are two dimensional. Efforts are undertaken to extend the numerical simulations to three dimensional space and to multiple particles.

\section{Acknowledgement}

The work presented here was supported in part by NSF through CMMI-1200075. Discussions with Professor Peter Voorhees at Northwestern University are gratefully acknowledged. 


\section{References}

Ardell, A.J., Nickolson, R.B., 1966. on the Modulated Structure of Aged Ni-Al Alloys: with an Appendix On the elastic interaction between inclusions by J. D. Eshelby. Acta Metallurgica, 1295-1309

Arora, J.S., 1993. An Exposition of the Material Derivative Approach for Structural Shape Sensitivity Analysis. Computer Methods in Applied Mechanics and Engineering 105, 41-62.

Belytschko, T., Moës, N., Usui, S., Parimi, C., 2001. Arbitrary discontinuities in finite elements. International Journal for Numerical Methods in Engineering 50, 993-1013.

Bordas, S., Nguyen, P.V., Dunant, C., Guidoum, A., Nguyen-Dang, H., 2007. An extended finite element library. Int J Numer Meth Eng 71, 703-732.

Braun, R.J., Mcfadden, G.B., Coriell, S.R., 1994. Morphological Instability in Phase-Field Models of Solidification. Physical Review E 49, 4336-4352.

Cahn, J.W., 1980. Surface stress and the chemical-equilibrium of small crystals .1. The case of the isotropic surface. Cc/Eng Tech Appl Sci 28, 1333-1338.

Cahn, J.W., Hilliard, J.E., 1958. Free Energy of a Nonuniform System .1. Interfacial Free Energy. J Chem Phys 28, 258-267.

Cermelli, P., Fried, E., Gurtin, M.E., 2005. Transport relations for surface integrals arising in the formulation of balance laws for evolving fluid interfaces. Journal of Fluid Mechanics 544, 339-351.

Chen, L.Q., 2002. Phase-field models for microstructure evolution. Annual Review of Materials Research 32, 113-140.

Chen, Z., Chu, K.T., Srolovitz, D.J., Rickman, J.M., Haataja, M.P., 2010. Dislocation climb strengthening in systems with immobile obstacles: Three-dimensional level-set simulation study. Phys Rev B 81.

Chessa, J., Smolinski, P., Belytschko, T., 2002. The extended finite element method (XFEM) for solidification problems. International Journal for Numerical Methods in Engineering 53, 1959-1977.

Conley, J.G., Fine, M.E., Weertman, J.R., 1989. Effect of Lattice Disregistry Variation on the Late Stage Phase-Transformation Behavior of Precipitates in Ni-Al-Mo Alloys. Acta Metallurgica 37, 1251-1263.

Cui, Z.W., Gao, F., Qu, J.M., 2012. A finite deformation stress-dependent chemical potential and its applications to lithium ion batteries. J Mech Phys Solids 60, 1280-1295.

Dingreville, R., Qu, J., 2009. A semi-analytical method to estimate interface elastic properties. Comp Mater Sci 46, 83-91. 
Dingreville, R., Qu, J.M., 2008. Interfacial excess energy, excess stress and excess strain in elastic solids: Planar interfaces. J Mech Phys Solids 56, 1944-1954.

Doi, M., 1996. Elasticity effects on the microstructure of alloys containing coherent precipitates. Progress in Materials Science 40, 79-180.

Doi, M., Miyazaki, T., Wakatsuki, T., 1984. The Effect of Elastic Interaction Energy on the Morphology of Gamma'-Precipitates in Nickel-Based Alloys. Materials Science and Engineering 67, 247-253.

Duan, H.L., Wang, J., Huang, Z.P., Karihaloo, B.L., 2005. Size-dependent effective elastic constants of solids containing nano-inhomogeneities with interface stress. J Mech Phys Solids 53, 1574-1596.

Duddu, R., Chopp, D.L., Voorhees, P., Moran, B., 2011. Diffusional evolution of precipitates in elastic media using the extended finite element and the level set methods. Journal of Computational Physics 230, 1249-1264.

Eshelby, J.D., 1975. Elastic Energy-Momentum Tensor. Journal of Elasticity 5, 321-335.

Fahrmann, M., Fratzl, P., Paris, O., Fahrmann, E., Johnson, W.C., 1995. Influence of Coherency Stress on Microstructural Evolution in Model Ni-Al-Mo Alloys. Acta Metallurgica Et Materialia 43, 1007-1022.

Fahrmann, M., Hermann, W., Fahrmann, E., Boegli, A., Pollock, T.M., Sockel, H.G., 1999. Determination of matrix and precipitate elastic constants in (gamma-gamma ') Ni-base model alloys, and their relevance to rafting. Mat Sci Eng a-Struct 260, 212-221.

Flory, P.J., 1941. Thermodynamics of high polymer solutions. Journal of Chemical Physics 9, 660-661.

Fries, T.P., 2008. A corrected XFEM approximation without problems in blending elements. International Journal for Numerical Methods in Engineering 75, 503-532.

Gao, X., Huang, Z.P., Qu, J.M., Fang, D.N., 2014. A curvature-dependent interfacial energy-based interface stress theory and its applications to nano-structured materials: (I) General theory. J Mech Phys Solids 66, 59-77.

Gurtin, M.E., 2000. Configurational forces as basic concepts of continuum physics. Springer, New York.

Gurtin, M.E., Murdoch, A.I., 1975. Continuum Theory of Elastic-Material Surfaces. Archive for Rational Mechanics and Analysis 57, 291-323.

Huggins, M.L., 1941. Solutions of long chain compounds. Journal of Chemical Physics 9, 440-440.

Isheim, D., Seidman, D.N., 2004. Nanoscale studies of segregation at coherent heterophase interfaces in alpha-Fe based systems. Surface and Interface Analysis 36, 569-574.

Jou, H.J., Leo, P.H., Lowengrub, J.S., 1997. Microstructural evolution in inhomogeneous elastic media. Journal of Computational Physics 131, 109-148. 
Larche, F., Cahn, J.W., 1973. Linear Theory of Thermochemical Equilibrium of Solids under Stress. Acta Metallurgica 21, 1051-1063.

Larche, F., Cahn, J.W., 1978. Non-Linear Theory of Thermochemical Equilibrium of Solids under Stress. Acta Metallurgica 26, 53-60.

Leo, P.H., Lowengrub, J.S., Nie, Q., 2000. Microstructural evolution in orthotropic elastic media. Journal of Computational Physics 157, 44-88.

Logg, A., Wells, G.N., 2010. DOLFIN: Automated Finite Element Computing. Acm Transactions on Mathematical Software 37.

Lou, Y., Bassani, J.L., 2008. Guided assembly of nanostructures via elastic interactions. J Mech Phys Solids 56, 3507-3526.

Ma, Y., Ardell, A.J., 2007. Coarsening of gamma (Ni-Al solid solution) precipitates in a gamma' (Ni3Al) matrix. Acta Materialia 55, 4419-4427.

Maraldi, M., Wells, G.N., Molari, L., 2011. Phase field model for coupled displacive and diffusive microstructural processes under thermal loading. Journal of the Mechanics and Physics of Solids 59, 1596-1612.

Miyazaki, T., Imamura, H., Kozakai, T., 1982. The Formation of Gamma'-Precipitate Doublets in Ni-Al Alloys and Their Energetic Stability. Materials Science and Engineering 54, 9-15.

Moelans, N., Blanpain, B., Wollants, P., 2008. An introduction to phase-field modeling of microstructure evolution. Calphad-Computer Coupling of Phase Diagrams and Thermochemistry 32, 268-294.

Osher, S., Fedkiw, R.P., 2002. Level set methods and dynamic implicit surfaces. Springer, New York.

Osher, S., Sethian, J.A., 1988. Fronts Propagating with Curvature-Dependent Speed - Algorithms Based on Hamilton-Jacobi Formulations. Journal of Computational Physics 79, 12-49.

Porter, D.A., Easterling, K.E., 1992. Phase transformations in metals and alloys, 2nd ed. Chapman \& Hall, London ; New York.

Povstenko, Y.Z., 1993. Theoretical Investigation of Phenomena Caused by Heterogeneous Surface-Tension in Solids. Journal of the Mechanics and Physics of Solids 41, 1499-1514.

Rodney, D., Le Bouar, Y., Finel, A., 2003. Phase field methods and dislocations. Acta Materialia 51, 17-30.

Sethian, J.A., 1996. Level set methods : evolving interfaces in geometry, fluid mechanics, computer vision, and materials science. Cambridge University Press, Cambridge.

Singer-Loginova, I., Singer, H.M., 2008. The phase field technique for modeling multiphase materials. Reports on Progress in Physics 71. 
Steinmann, P., Maugin, G.A., 2005. Mechanics of material forces. Springer, New York.

Su, C.H., Voorhees, P.W., 1996a. The dynamics of precipitate evolution in elastically stressed solids .1. Inverse coarsening. Acta Materialia 44, 1987-1999.

Su, C.H., Voorhees, P.W., 1996b. The dynamics of precipitate evolution in elastically stressed solids .2. Particle alignment. Acta Materialia 44, 2001-2016.

Swaminathan, N., Qu, J., Sun, Y., 2007. An electrochemomechanical theory of defects in ionic solids. I. Theory. Philosophical Magazine 87, 1705-1721.

Thornton, K., Akaiwa, N., Voorhees, P.W., 2001. Dynamics of late-stage phase separation in crystalline solids. Physical Review Letters 86, 1259-1262.

Tonks, M.R., Gaston, D., Millett, P.C., Andrs, D., Talbot, P., 2012. An object-oriented finite element framework for multiphysics phase field simulations. Computational Materials Science 51, 20-29.

Voorhees, P.W., Mcfadden, G.B., Johnson, W.C., 1992. On the Morphological Development of second-Phase Particles in Elastically-Stressed Solids. Acta Metallurgica Et Materialia 40, 2979-2992.

Wang, S.L., Sekerka, R.F., 1996. Algorithms for phase field computation of the dendritic operating state at large supercoolings. Journal of Computational Physics 127, 110-117.

Wang, Y., Khachaturyan, A.G., 1995. Shape Instability during Precipitate Growth in Coherent Solids. Acta Metallurgica Et Materialia 43, 1837-1857.

Wodo, O., Ganapathysubramanian, B., 2011. Computationally efficient solution to the Cahn-Hilliard equation: Adaptive implicit time schemes, mesh sensitivity analysis and the 3D isoperimetric problem. Journal of Computational Physics 230, 6037-6060.

Zabaras, N., Ganapathysubramanian, B., Tan, L.J., 2006. Modelling dendritic solidification with melt convection using the extended finite element method. Journal of Computational Physics 218, 200-227.

Zaeem, M.A., Mesarovic, S.D., 2010. Finite element method for conserved phase fields: Stress-mediated diffusional phase transformation. Journal of Computational Physics 229, 9135-9149.

Zhao, X.J., Bordas, S.P.A., Qu, J.M., 2013a. A hybrid smoothed extended finite element/level set method for modeling equilibrium shapes of nano-inhomogeneities. Computational Mechanics 52, 1417-1428.

Zhao, X.J., Duddu, R., Bordas, S.P.A., Qu, J.M., 2013b. Effects of elastic strain energy and interfacial stress on the equilibrium morphology of misfit particles in heterogeneous solids. Journal of the Mechanics and Physics of Solids $61,1433-1445$. 
Zhao, X.J., Qu, J., 2012. Effects of interfacial excess energy on the elastic field of a nano-inhomogeneity. Mech Mater 55, 41-48.

Zhao, X.J., Rajapakse, R.K.N.D., 2009. Analytical solutions for a surface-loaded isotropic elastic layer with surface energy effects. Int J Eng Sci 47, 1433-1444. 